\title{
comportement mécanique des sols injectés aux produits chimiques
}

par

\author{
P. Luong \\ Ingénieur ENPC \\ Docteur ès Sciences
}

Laboratoire de Mécanique des Solides de l'Ecole Polytechnique

M. Gandais

Ingénieur AM

MS Mécanique des Sols (Berkeley)

Laboratoire d'Essais et Mesures

de la Société SIF-Bachy

et

\section{P. Allemand}

Ingénieur ICPI

Division Chimie fine

de la Société Rhône-Poulenc

\section{COMPORTEMENT MECANIOUE DES SOLS INJECTES AUX PRODUITS CHIMIQUES}

Le comportement mécanique d'un sable fin consolidé par injection de gels de silicates ou de résines acrylamides est étudié à partir d'essais comprenant: la compression simple avec vitesses de déformation ou de charge contrôlées, la relaxation, le fluage et le fluage sous effet de poinçonnement.

La résistance mécanique est liée au facteur temps qui intervient dans le mode de mise sous contraintes et dans la durée d'application des contraintes.

L'amplitude et la variation dans le temps de la résistance mécanique sont étroitement liées au dosage interne des différents constituants des produits injectés.

Ces notions permettent d'améliorer et de rendre sûr le choix des produits à injecter suivant que le traitement envisagé a un caractère provisoire ou définitif.
MECHANICAL BEHAVIOUR OF GROUTED SOILS WITH CHEMICAL PRODUCTS

The mechanical performance of a fine sand consolidated by silicate gels or acrylamide resins grouting is studied through tests including: the simple compression with controlled rates of deformation or of load, relaxation, creep or creep as an effect of punching.

The mechanical strength is linked to the time factor which intervenes in the manner of application of stress and in the duration of application of stresses.

The amplitude and the variation in time of the mechanical strength are closely linked to the internal batching of the different constituents of the products injected.

The concepts make it possible to improve and to give reliability to the choice of the products to be grouted, depending on whether the treatment considered has a provisional or a final character. 
Comité Français de Mécanique des Sols

Institut technique des Bâtiments et des Travaux Publics 


\section{comportement mécanique des sols injectés aux produits chimiques}

par P. LUONG, M. GANDAIS et P. ALLEMAND

Allocution du Président BOUGARD

Tout d'abord, je voudrais excuser M. Lupiac, qui devait présider cette conférence. Effectivement, il a été appelé sous d'autres cieux pour discuter avec d'éventuels clients, ce qui est assez original pour un maître d'œuvre, et c'est la raison pour laquelle je suis ici.

Vous savez que M. Lupiac est directeur des Travaux Neufs de la RATP, et Président de l'Association Française des Travaux en Souterrain. Il était donc désigné à double titre pour présider cette séance.

La conférence qui va vous être présentée porte sur le comportement mécanique des sols traités au moyen d'injections de produits chimiques. C'est en fait le résultat actuel de recherches originales et d'essais qui ont été menés par les trois jeunes ingénieurs qui sont ici: M. Luong qui va faire l'exposé, M. Gandais et M. Allemand,

Comme vous allez le voir, la qualité de ces travaux ne pouvait être qu'excellente si l'on en juge d'après les références de ces trois ingénieurs.

M. LuONg est ingénieur civil des Ponts et Chaussées. Il est entré en 1962 au Laboratoire de mécanique des solides à l'Ecole Polytechnique, il est devenu Docteur ès Sciences en 1965 et il est maître de conférences à l'ENCREF.

M. GandaIs est ingénieur des Arts et Métiers. Il est allé à l'Université de Berkeley pour faire un " master of sciences " en mécanique des sols. Depuis, il est entré à la Société Bachy où il dirige le laboratoire " essais-mesures " et où il a mis au point des gels de silice avec réactif organique et des résines.

Enfin, M. Allemand est un ancien élève de l'Institut de chimie physique industriel de Lyon. Il est rentré à la Société Progil en 1968. Depuis, il y a eu le groupement avec Rhône-Poulenc et il a été rattaché à la division chimie fine où il travaille sur les produits chimiques destinés au génie civil et aux travaux publics.

M. LuONG va parler au nom des trois et MM. ALLEMAND et GANDAIs répondront à vos questions à la fin de l'exposé.

\section{EXPOSE DE P. LUONG, M. GANDAIS, P. ALLEMAND}

Cette étude présente un résumé des résultats expérimentaux d'une recherche coordonnée entre les Sociétés SIF-Bachy et Rhône-Poulenc et effectuée au Labora- toire de Mécanique des Solides de l'Ecole Polytechnique. Le sujet en est le comportement mécanique des terrains granulaires fins à très fins, traités par injections de produits chimiques.

\section{TECHNIQUE DE L'INJECTION DES SOLS GRANULAIRES FINS}

\subsection{Principe général}

La construction, la modification ou l'entretien d'ouvrages de génie civil nécessite souvent une amélioration des caractéristiques des sols.

Cette amélioration, dans la plupart des cas, porte sur les points suivants :

1) réduction de la perméabilité (étanchement);

2) amélioration de la résistance mécanique (consolidation).

Ces buts différents n'impliquent pas forcément des traitements spécifiques séparés et, bien souvent, les sols feront l'objet d'un traitement simultané lors d'une même opération d'injection. Cependant, l'étude réalisée ici porte uniquement sur le problème plus délicat de la consolidation.
L'effet de consolidation par injection d'un sol granulaire s'obtient en remplissant les vides du milieu à l'aide d'un coulis qui, fluide au moment de sa mise en place, va être le siège d'une réaction chimique conduisant à sa rigidification. Le coulis est mis en place sous pression, à partir de forages, et après rigidification, il se présente comme une matrice qui noie et solidarise les grains de la formation traitée. Les squelettes granulaires concernés sont constitués généralement par des alluvions sablo-graveleuses, des sables ou des sables silteux qui présentent une perméabilité d'interstice de $10^{-4} \mathrm{~m} / \mathrm{s}$ à $10^{-6} \mathrm{~m} / \mathrm{s}$. Il est donc demandé au coulis de conférer, à l'ensemble matrice-squelette, des caractéristique géotechniques sur lesquelles le projeteur pourra compter. Ces caractéristiques vont conditionner le traitement. 


\subsection{Conditions de traitement}

\subsection{Critère de qualité}

La qualité du traitement à réaliser entraîne, pour le spécialiste en injection, la définition des moyens matériels et techniques à utiliser mais surtout le choix du ou des produits à mettre en œuvre. Cette qualité de traitement est caractérisée de façon courante par le critère de la résistance à la compression simple. En effet, les praticiens de l'injection ont eu besoin, très tôt, d'un critère pour établir une classification des divers produits utilisables et, par application de la résistance des matériaux classique, le choix s'est porté sur la valeur de la résistance à la compression simple d'échantillons de sable consolidé par le produit d'injection considéré. Ce critère est entré suffisamment dans la pratique courante pour que, d'une part on le retrouve comme élément principal dans les spécifications techniques fixées par certains maîtres d'ouvrages, et d'autre part pour que de nombreux projeteurs l'utilisent pour tenter de justifier les calculs de stabilité des ouvrages.

Malheureusement, on constate que la résistance à la compression simple $\mathrm{R}_{c}$ des sols traités avec des produits d'injection d'utilisation courante, dépend du facteur temps d'une façon très significative. Quelques rares publications telles que celles de J. Warner et de Ch. Kutzner ont commencé à porter ce phénomène à la connaissance générale. Il fallait donc poursuivre et essayer d'aller plus loin pour se rendre compte que le critère $R_{c}$ utilisé habituellement est une fonction qui dépend de façon importante de la durée de mise sous contrainte ; il en ressort que le critère $\mathrm{R}_{c}$ ne peut plus être considéré comme le seul paramètre à prendre en compte dans le calcul des ouvrages. Le contenu de l'expérimentation présentée ci-après a donc été l'étude de l'influence du facteur temps dans le comportement mécanique d'un sol fin injecté, sous diverses conditions de charges et de déformations, afin de permettre la définition des meilleures conditions d'emploi des coulis utilisés.

\subsection{Choix des produits}

\subsection{Conditions générales}

S'agissant de traiter des matériaux fins à très fins, le spécialiste de l'injection va se trouver confronté à la tâche délicate de choisir le ou les produits qui lui permettront de réaliser le traitement de consolidation de façon satisfaisante. Il va, pour cela, tenir compte de quatre critères principaux, communs à tous les traitements par injection. La tâche se complique parfois par l'intervention de paramètres secondaires propres à des conditions d'environnement très particulières (température, eaux à teneurs inhabituelles en produits néfastes pour les coulis, eaux en mouvement au voisinage de rabattements de nappes, etc.). Ces conditions qui donnent aux travaux à réaliser la nature de cas particuliers, n'entrent pas dans le cadre de cette étude.

Le choix du produit est donc guidé par :

a) la viscosité et son évolution

Cette viscosité devra être la plus faible possible et pendant un laps de temps le plus long possible pour que l'imprégnation de matériaux très fins puisse se faire de façon homogène d'une part, et dans des délais acceptables d'autre part.

\section{b) le temps de prise}

Celui-ci doit pouvoir être réglé à une durée suffisamment longue pour, justement, laisser le temps à l'opérateur de procéder à l'imprégnation des terrains fins. La gamme des temps de prise la plus couramment utilisée s'étale d'une quarantaine à une centaine de minutes.

\section{c) la résistance mécanique escomptée}

Ce point n'est évidemment qu'un rappel du critère de qualité retenu dans la pratique courante.

d) le compromis économie - efficacité - commodité d'emploi

Les produits d'injection retenus doivent enfin permettre de résoudre les différents problèmes d'injection de façon satisfaisante et dans les meilleures conditions de sécurité, pour un coût acceptable compte tenu des résultats attendus.

\subsection{Nature des produits}

L'examen des quatre paramètres précédents conduit à une constatation : il n'existe qu'un nombre restreint de familles de produits susceptibles de les satisfaire et d'être compatibles avec la plupart des terrains rencontrés.

La première famille, et la plus largement utilisée dans le monde, est celle des gels à base de silicates de soude. Elle a été retenue pour cette étude.

La deuxième famille importante est celle des résines organiques en phase aqueuse. Parmi celles-ci, les résines acrylamides sont les plus couramment utilisées dans la pratique des injections. Elles ont été incluses également dans cette étude.

Les deux types de produits étudiés comportent chacun plusieurs constituants dont les dosages sont variables et précisés par le spécialiste en injection en fonction du degré de qualité du traitement à réaliser. Ces dosages sont définis par des valeurs attribuées à différents paramètres caractéristiques des compositions.

\section{a) Gels de silicate}

Les paramètres retenus pour caractériser la composition d'un gel par unité de volume, sont :

la teneur en réactif : $\alpha=\frac{\text { poids de réactif }}{\text { volume de silicate }}$

la dilution du silicate : $\beta=\frac{\text { volume d'eau }}{\text { volume de silicate }}$

le taux de neutralisation : $\mathrm{N} \%$

L'utilisation du taux de neutralisation $\mathrm{N} \%$, qui est lié de façon linéaire à la valeur de $\alpha$ pour un réactif donné, nécessite un commentaire.

Il y a encore peu de temps, on pouvait constater en laboratoire mais également sur chantier, que les gels de silicate avaient un comportement médiocre, ou parfois franchement mauvais, lorsqu'ils étaient immergés dans l'eau (travaux sous la nappe). Il était donc très délicat de s'engager dans la réalisation de travaux de consolidations à long terme avec de tels produits. La situation est beaucoup plus claire aujourd'hui.

De façon très schématique, rappelons qu'un silicate de soude en solution acqueuse se transforme en un gel, plus ou moins résistant, par rupture de l'équilibre interne silice-soude. Cette rupture s'obtient, dans le cas de l'utilisation de réactifs organiques du type esters, par élimination d'une certaine quantité de soude par neutralisation avec les acides apportés par le réactif.

Des études en laboratoire ont montré de façon indiscutable que la pérénité des gels immergés dans l'eau était directement liée au taux de neutralisation. Il n'y a pas encore très longtemps, la progression 
des études était bloquée par l'absence de réactifs qui auraient permis d'atteindre des taux de neutralisation élevés tout en conservant des temps de prise suffisamment longs pour que de tels gels soient utilisables.

Cette carence a été levée avec la venue, sur le marché, des durcisseurs de la série 600 , fabriqués par la Société Rhône-Poulenc; ces durcisseurs permettent de préparer, de façon simple, des gels ayant d'une part les temps de prise pratiques voulus, et d'autre part au taux de neutralisation souhaité, et ce jusqu'à $100 \%$. De ce fait, il est devenu possible de mettre en œuvre des gels ayant une excellente tenue dans l'eau. Il semblait logique que cetté stabilité de nature chimique s'accompagne d'une certaine stabilité des propriétés mécaniques. Pour cette raison, le taux de neutralisation $\mathrm{N} \%$ fait partie des paramètres caractéristiques d'une composition qui ont été retenus dans cette étude.

\section{b) Résines acrylamides}

Ces résines sont utilisées fréquemment, dans le monde, pour résoudre des problèmes d'étanchement par injections. Elles confèrent, une certaine résistance mécanique aux terrains traités. Elles se présentent sous la forme de solutions aqueuses et la polymérisation se contrôle aisément par l'addition de catalyseurs au moment de l'utilisation. Les teneurs en catalyseurs, qui ne sont propres qu'à la phase de mise en œuvre ne constituent pas un paramètre principal caractérisant la composition.

Deux facteurs principaux permettent de faire varier la résistance mécanique apportée par ces résines. Il s'agit de :

1) l'extrait sec pondéral de la solution au moment de l'injection; il est exprimé en ES \% ;

2) la teneur pondérale d'un réticulant supplémentaire qui peut être additionné à la résine au moment de la fabrication; elle est exprimée en TR \%.

Ces deux paramètres sont utilisés dans cette étude.

Un commentaire est nécessaire pour expliquer le choix de ce type de résine dans une étude qui porte sur les problèmes de consolidation; en effet, elles n'apportent, en ce qui concerne les critères utilisés jusqu'à présent (compression simple), que des résistances mécaniques assez faibles.

La raison de leur emploi est simple et logique : remarquant que certains gels, très performants d'après le test de compression simple, se dégradaient de façon considérable lorsque les modalités de mise sous contraintes faisaient intervenir le facteur temps, on pouvait se demander si ce type de résine, bien que rejeté d'après le critère de qualité classique en raison de leur faible résistance à la compression, n'était finalement pas plus intéressant et plus sûr que certains gels de silicate pour résoudre certains problèmes de consolidations définitives.

\section{METHODOLOGIE EXPERIMENTALE}

\subsection{Matériaux et produits}

\subsection{Squelette granulaire}

S'agissant d'étudier les caractéristiques géotechniques de l'ensemble squelette-matrice tout en modifiant la nature et les dosages de la matrice, il fallait que la partie granulaire conserve des caractéristiques constantes pour tous les essais.

Le choix s'est porté sur le sable utilisé le plus couramment dans les quelques laboratoires qui procèdent à des études et recherches et dont l'utilisation est recommandée par l'Association Française des Travaux en Souterrains (AFTES). Il s'agit du sable de Fontainebleau commercialisé sous l'appellation MN 30. C'est un sable fin, blanc, siliceux à grains très résistants et anguleux.

Les caractéristiques de la répartition granulométrique sont :

$$
\begin{aligned}
& d_{100}=0.35 \mathrm{~mm} \quad d_{60}=0.23 \mathrm{~mm} \\
& d_{10}=0.14 \mathrm{~mm} \quad d_{0}=0.08 \mathrm{~mm}
\end{aligned}
$$

Le coefficient de Hazen vaut environ : $\frac{d_{10}}{d_{60}} \simeq 1.6$

\subsection{Produits injectés constitutifs de la matrice}

a) Gels de silicates

Silicate : silicate de soude de rapport pondéral : Si $02 / \mathrm{Na}_{2} \mathrm{O}=3.3$

Densité : 1.33 (35 à $37^{\circ}$ Baumé).

Réactifs: Durcisseurs de la série 600 (RhônePoulenc).

Il s'agit de mélanges d'esters méthyliques et éthyliques de diacides. Les dosages internes peuvent être modifiés à volonté pour atteindre un taux de neutralisation $\mathrm{N} \%$ tout en conservant un temps de prise déterminé: Suivant les dosages internes, les réactifs sont désignés par $\mathrm{B}, \mathrm{C}$ et $\mathrm{E}$ dans cette étude.

Dosages des gels de silicate utilisés : ils sont au nombre de douze et englobent la totalité des dosages utilisés dans la pratique courante. Ces dosages sont visualisés sur la figure 1 : ils présentent tous un temps de prise de $45 \mathrm{mn}$ à $20^{\circ} \mathrm{C}$.

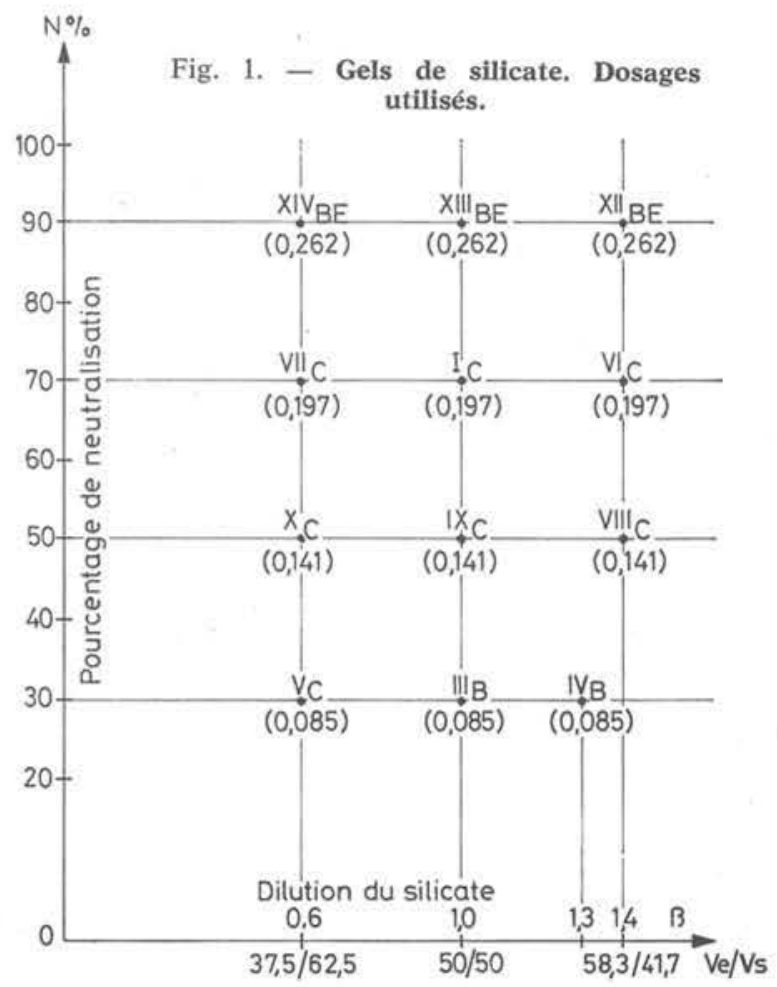




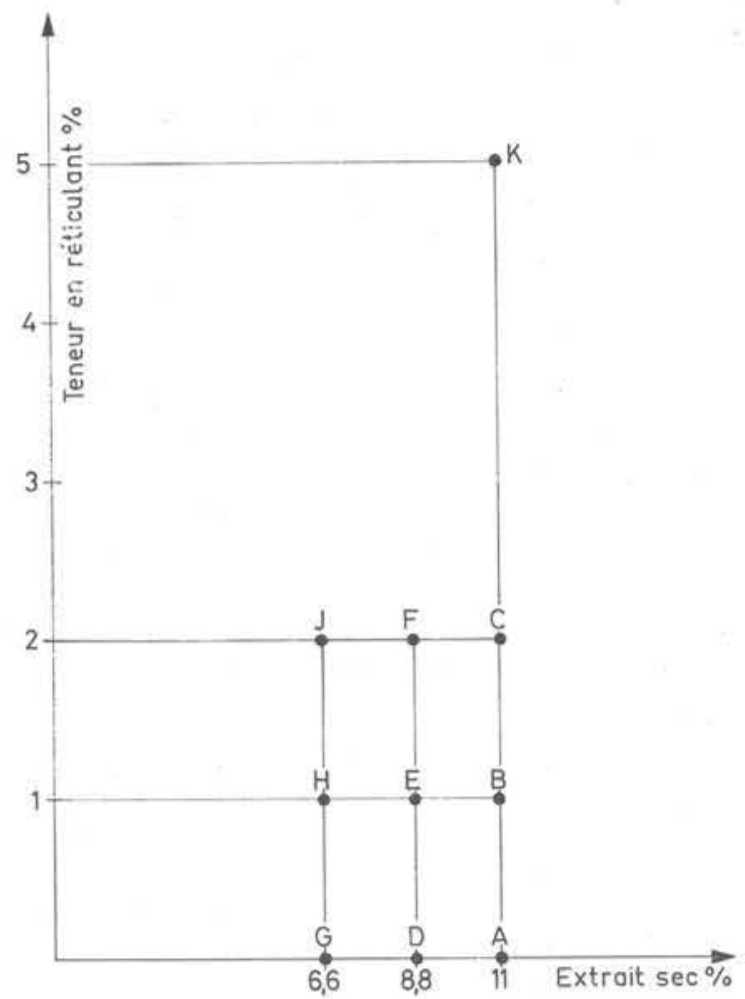

Fig. 2. - Résine acrylamide. Dosages utilisés.

\section{b) Résine acrylamide}

Résine: Résine acrylamide en solution aqueuse commercialisée sous l'appellation Rocagil 1295 (Rhône-Poulenc).

Reticulant: Méthylènebisacrylamide (M.B.A.) de la même société.

Dosages utilisés : ils sont au nombre de dix et visualisés sur la figure 2 .

Les teneurs en catalyseurs ont été réglées pour que tous ces coulis aient un temps de prise de $45 \mathrm{mn}$ à $20^{\circ} \mathrm{C}$.

\subsection{Préparation des éprouvettes pour essais}

Les éprouvettes sont préparées par injection des coulis à l'intérieur de colonnes de sable préalablement saturées d'eau. Les opérations se déroulent de la façon suivante.

\subsection{Préparation des colonnes}

Le sable est mis en place dans des tubes verticaux en P.V.C. de $1 \mathrm{~m}$ de longueur et de diamètre intérieur $\varnothing i=42 \mathrm{~mm}$. Le sable est arrêté aux deux extrémités du tube par des obturateurs auto-bloquants qui ne permettent aucune expansion ou foisonnement du sable lors des opérations ultérieures.

La densité sèche en place du sable est de 1.70.

\subsection{Injection des colonnes}

Les colonnes de sable sont saturées d'eau par injection d'eau à partir du bas à l'aide d'une petite pompe à piston.

Les coulis sont préparés par malaxage et, dans le cas de gels de silicate, maintenus en agitation lente par un petit agitateur magnétique qui figure les malaxeurs de reprise installés sur les chantiers.

Lorsqu'une colonne est saturée d'eau, l'aspiration de la pompe est branchée sur le réservoir de coulis. Le coulis est ainsi injecté à partir du bas, en repoussant l'eau qui se trouve dans les interstices du sable (fig. 3).

Lorsque le coulis atteint le sommet de la colonne, l'injection est arrêtée et les orifices d'entrée et de sortie sont obturés. La pompe utilisée est à débit variable, réglable en cours de fonctionnement et les pressions d'injection sont de $0.1,0.2$ ou $0.3 \mathrm{MPa}$.

La saturation préalable à l'eau semble indispensable car elle représente le mieux ce qui se passe lors des traitements de terrain par injection. Les terrains parfaitement secs n'existent pratiquement pas et les coulis entrent toujours en contact avec les grains des formations traitées par l'intermédiaire de surfaces soit humides, soit franchement noyées lors des traitements sous nappe phréatique.

\subsection{Eprouvettes pour essais}

Les colonnes, obturées à leurs deux extrémités, sont conservées ainsi pendant sept jours pour que les phénomènes de prise soient stabilisés. L'ensemble squelette-matrice se trouve ainsi à l'abri de l'air et de toute dessication ou drainage pendant cette période.

Juste avant de procéder à des essais, les colonnes sont tronçonnées et chacune fournit dix échantillons de $85 \mathrm{~mm}$ de hauteur et de $42 \mathrm{~mm}$ de diamètre.

\subsection{Mode opératoire des essais effectués}

Les essais réalisés sont de divers types et se résument ainsi :

a) essais de compression simple de deux types :

- avec vitesse de déformation contrôlée $\dot{\varepsilon}=$ cte ;

- avec vitesse de charge contrôlée $\quad \dot{\sigma}=$ cte;

b) essais de relaxation ;

c) essai de fluage de longue durée sous charge statique ;

d) essai de poinçonnement.

\subsection{Essais de compression simple}

a) Vitesse de déformation contrôlée

Ces essais sont réalisés sur une presse Instron (fig. 4). Le déplacement du plateau dynamomètre est contrôlé et permet de réaliser la condition

$$
\dot{\varepsilon}=\frac{\Delta \mathrm{H}}{\mathrm{H}} / t=\text { cte. }
$$

\section{b) Vitesse de charge contrôlée}

Ces essais sont réalisés sur une presse hydraulique Mohr et Federhaff. Le déplacement du plateau est également contrôlé et permet de réaliser la condition

$$
\dot{\sigma}=\frac{\Delta \mathrm{F}}{\mathrm{S}} / \mathrm{t}=\text { cte. }
$$

Dans les deux cas, la déformation transversale $\varepsilon_{2}=\varepsilon_{3}$ a été mesurée par l'intermédiaire d'une ceinture équipée de jauges de déformation (fig. 5). Cette ceinture est située sur la circonférence centrale de l'éprouvette et maintenue par deux petits anneaux de caoutchouc qui n'exercent pas d'effort significatif sur l'éprouvette.

Les déformations longitudinales $\varepsilon_{1}$ et transversales $\varepsilon_{2}=\varepsilon_{3}$ ont été enregistrées en fonction de la charge. 


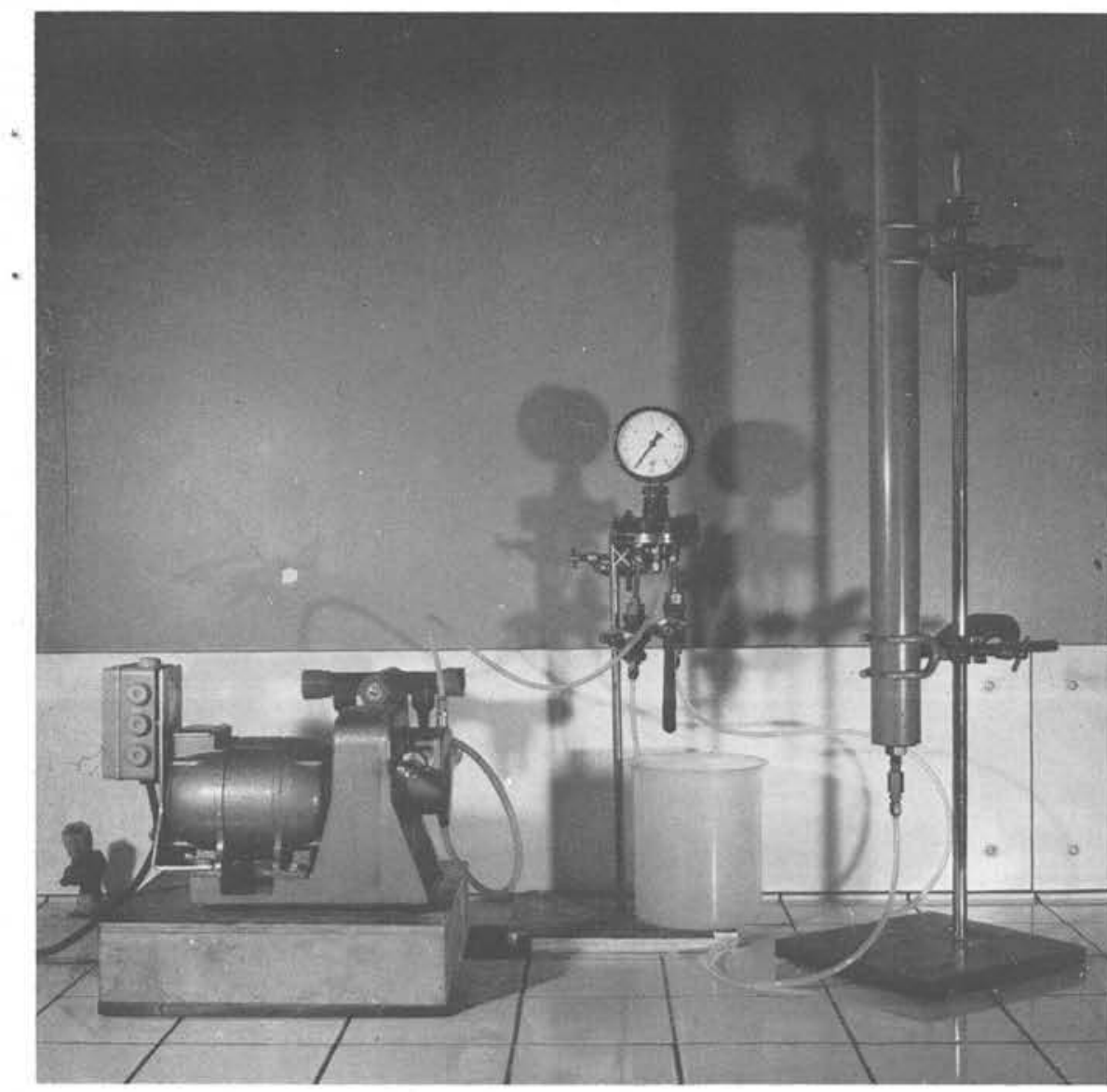

Fig. 3. - Injection du coulis.

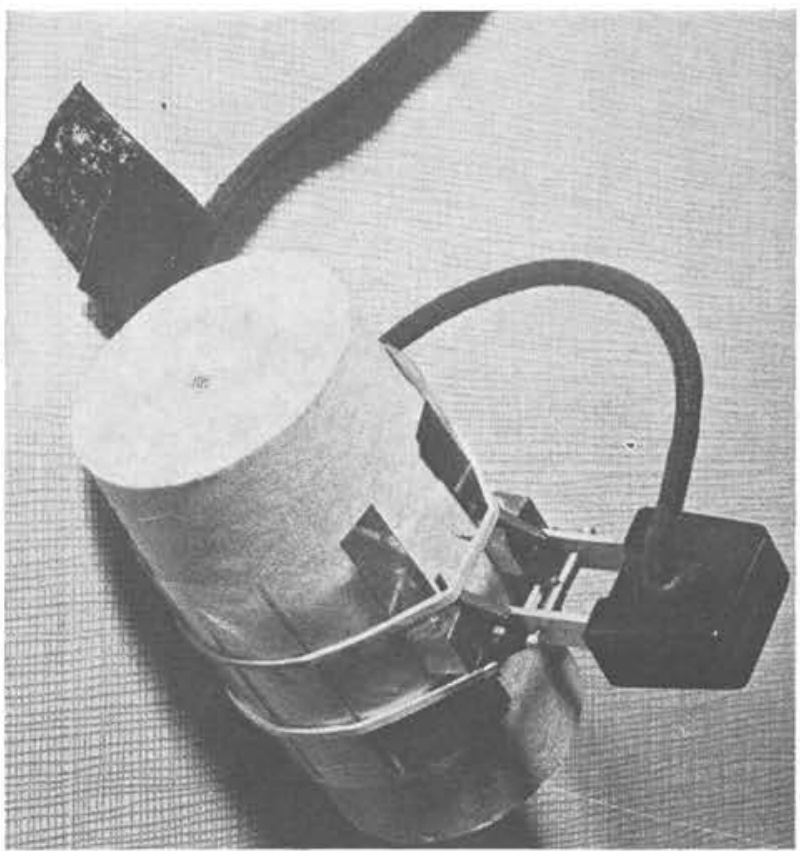

Fig. 5. - Mesure de la déformation transversale.

Fig. 4. - Presse d'essais à vitesse de déplacement contrôlée. 
Elles ont donc permis de suivre l'évolution de la variation relative de volume des éprouvettes $\frac{\Delta V}{V}$ au cours de la charge, et jusqu'à la rupture

$$
\frac{\Delta \mathrm{V}}{\mathrm{V}}=\varepsilon_{1}+2 \varepsilon_{2}
$$

où

$$
\varepsilon_{1}=\frac{\Delta H}{H} \text { est défini par le reccourcissement relatif }
$$

à la hauteur de l'éprouvette et,

$\varepsilon_{2}=\frac{\Delta(2 \pi R)}{2 \pi R}$ est égal à l'allongement relatif de la circonférence dans la zone centrale de l'éprouvette. Cette valeur est légèrement en excès si l'on tient compte de l'influence des extrémités.

\subsection{Essais de relaxation}

Quelques essais de relaxation ont été réalisés pour des charges correspondant en principe à $0.5 \mathrm{R}_{c}$; $0.75 \mathrm{R}_{c} ; 0.9 \mathrm{R}_{c}$, puis à la rupture complète.

Pour ces essais, l'éprouvette est mise en charge avec une vitesse de déformation axiale $\dot{\varepsilon}_{1}$ fixée et constante, jusqu'à une valeur fixée de la contrainte axiale $\sigma_{1}$. Le plateau de la presse est arrêté et l'évolution de $\sigma_{1}$ est enregistrée en fonction du temps.

Seuls quelques essais ont été réalisés, dans le but de connaître les divers aspects du phénomène. Leur nombre a été volontairement limité pour deux raisons :

1) le phénomène de relaxation n'intervient pratiquement jamais dans les traitements de consolidation de terrains par injection;
2) du point de vue expérimental, l'essai de relaxation interdit l'utilisation, pour d'autres tâches, d'un matériel important (presse) pendant un temps qui peut être très long.

\subsection{Essais de fluage}

Ces essais sous charge statique ont été menés pour de longues durées, jusqu'à trois mois.

L'éprouvette est soumise à une charge constante $\sigma_{1}$ dans un bâti de fluage et la déformation axiale $\varepsilon_{1}$ est donnée par la moyenne des lectures sur trois comparateurs précis à $\frac{1}{100} \mathrm{~mm}$ (fig. 6).

Les éprouvettes sont protégées de la dessication par une couche mince de paraffine.

\subsection{Essais de poinçonnement}

Les essais de poinçonnement ont été réalisés pour étudier l'influence d'une distribution non uniforme des contraintes vis-à-vis du fluage de ces matériaux (fig. 7).

Les échantillons, protégés également de la dessication par une pellicule de paraffine, sont montés dans des bâtis spéciaux. Dans ce cas, les éprouvettes ont une hauteur de $50 \mathrm{~mm}$. Des charges fixées et constantes sont appliquées sur des poinçons dont les sections de base sont de $0.5 \mathrm{~cm}^{2}$ et $1 \mathrm{~cm}^{2}$. L'enfoncement des poinçons est suivi dans le temps à l'aide de comparateurs précis au $\frac{1}{100} \mathrm{~mm}$.

Fig. 6. - Bâtis de fluage.

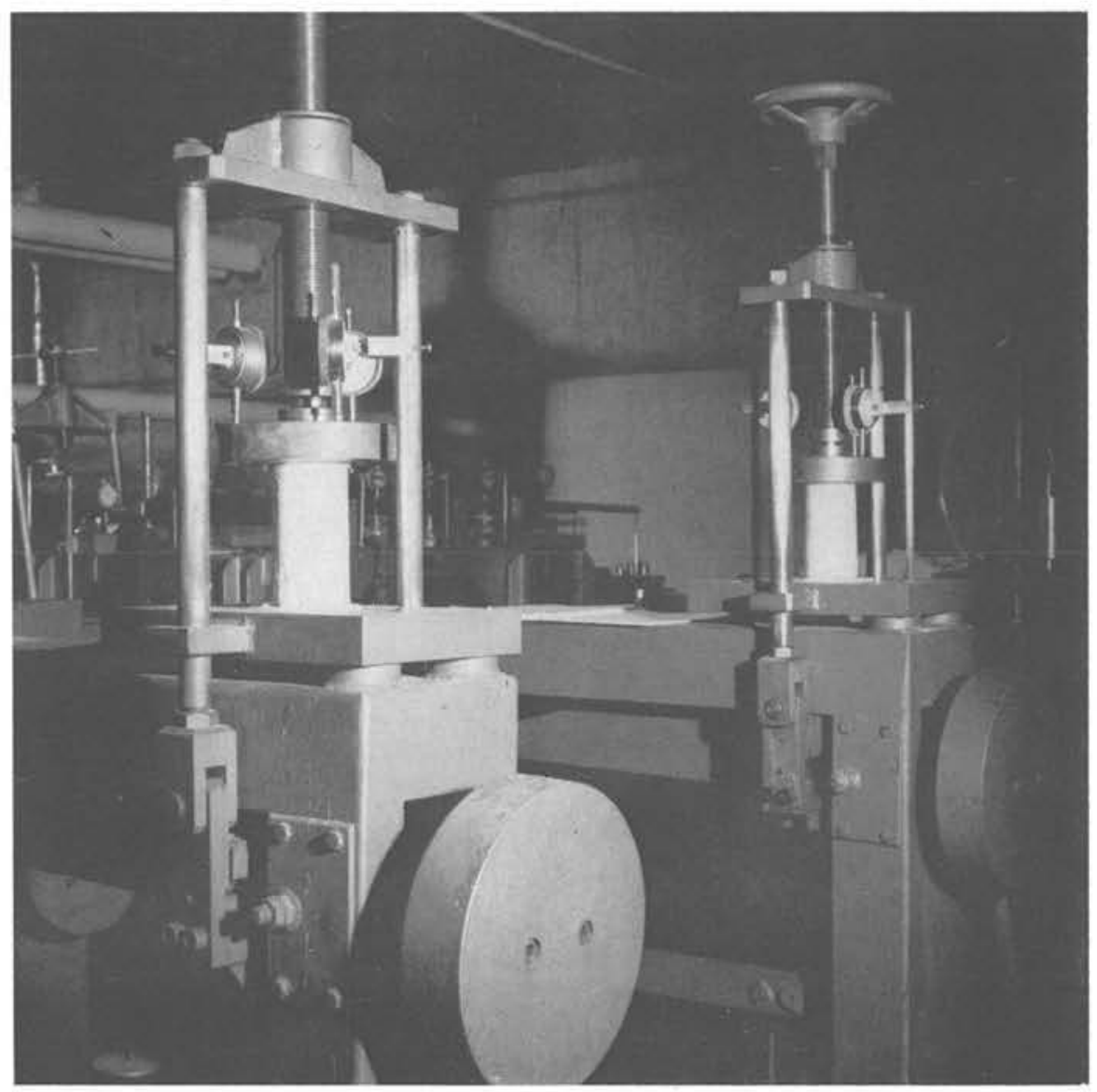


Fig. 7. - Essais de poinçonnement sous charge statique.

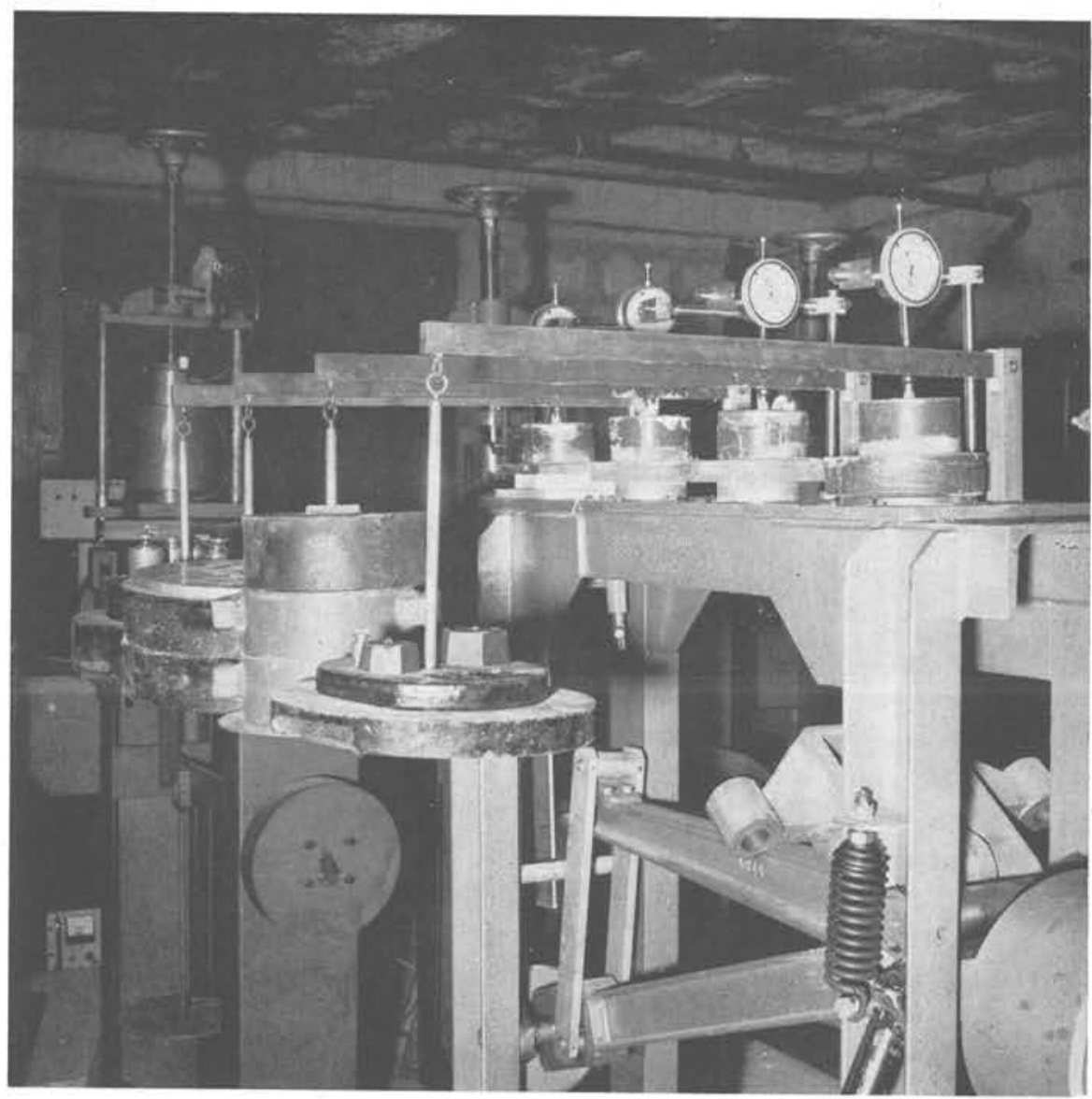

\section{CONSTATATIONS EXPERIMENTALES SUR LES SABLES INJECTES AUX GELS DE SILICATE}

\subsection{Variation de volume au cours de la mise sous contrainte}

Les figures 8 et 9 montrent les résultats obtenus lors d'essais de compression simple réalisés sur des éprouvettes injectées avec deux dosages de gels différents.
La première série (fig. 8) a été réalisée avec $\varepsilon_{1}$ contrôlé allant de $2 \cdot 10^{-6} / \mathrm{s}$ à $10^{-2} / \mathrm{s}$. La dernière série (fig. 9) s'est faite avec vitesse de charge $\sigma$ contrôlée allant de $0.12 \mathrm{kPa} / \mathrm{s}$ à $180 \mathrm{kPa} / \mathrm{s}$.

Il y est mis en évidence une diminution relative de volume au début du chargement. Il semble qu'il y ait une limite de résistance stable véritable relative à

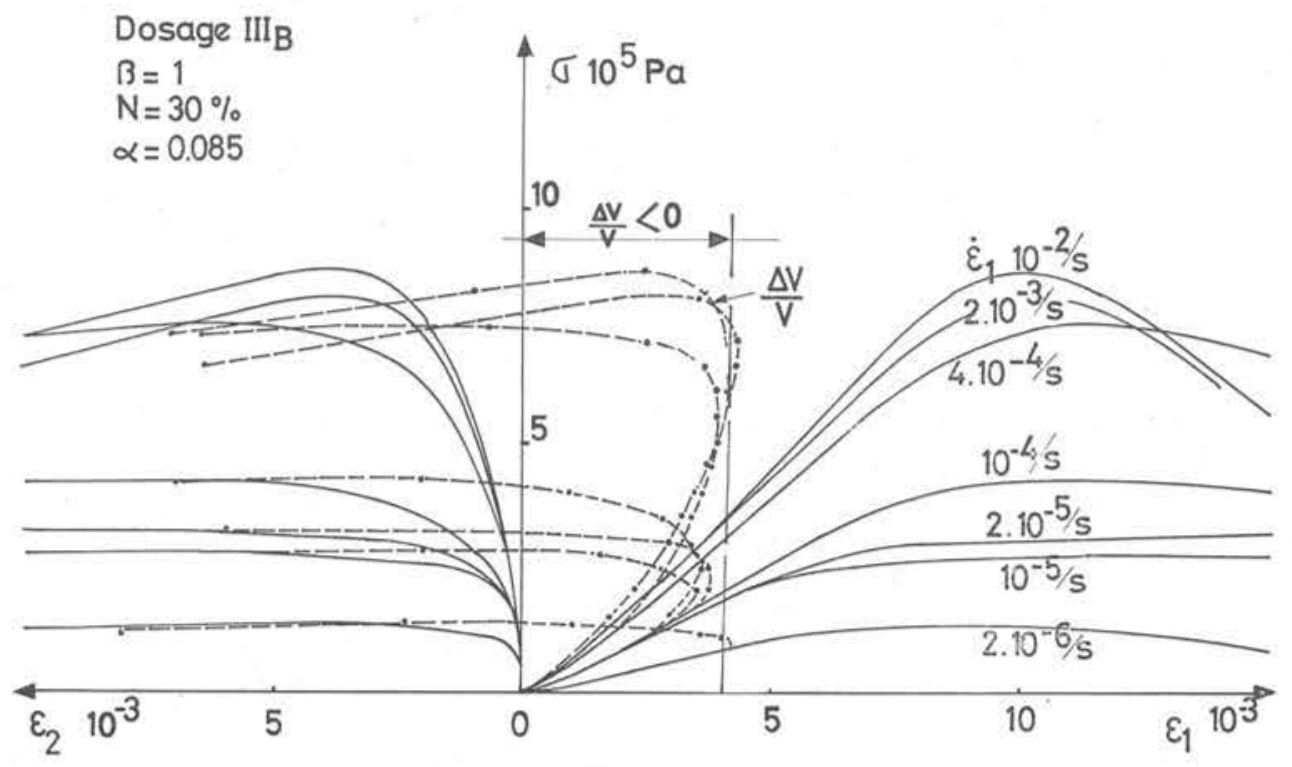

Fig. 8. - Compression simple avec vitesse de déformation $\dot{\varepsilon}_{1}$ contrôlée. Dosage $\operatorname{III}_{B}$. 


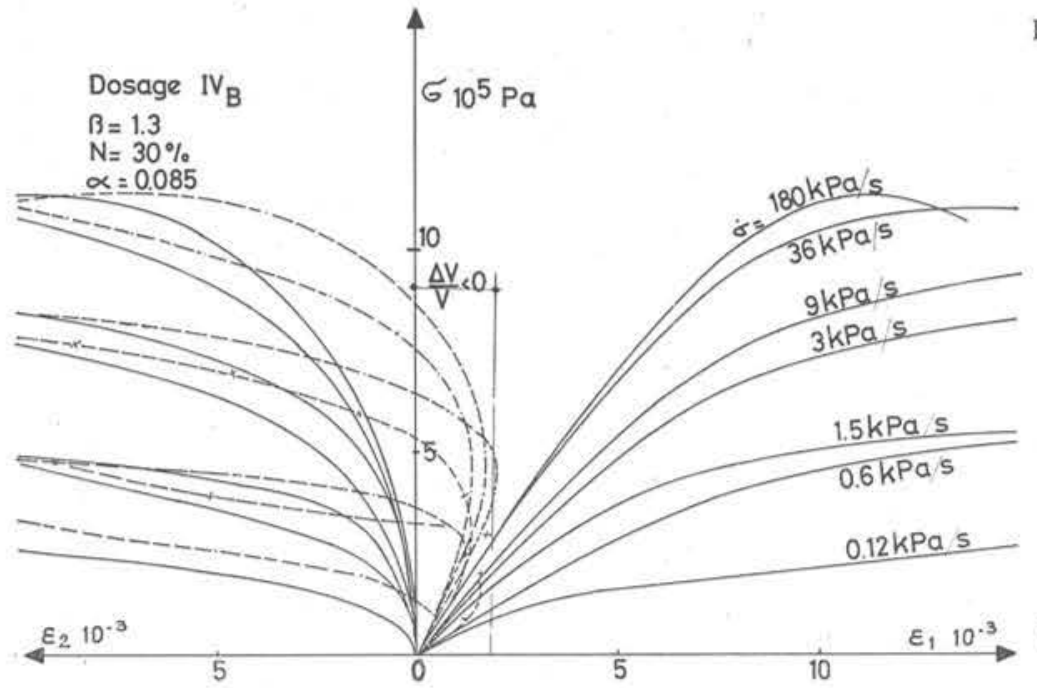

la variation de volume, au-delà de laquelle apparaît brusquement la dilatance.

Pour des charges inférieures à cette limite, on constate une diminution de volume $\frac{\Delta V}{V}=\varepsilon_{1}+2 \varepsilon_{2}<0$ jusqu'à une certaine valeur $\frac{\Delta V}{V}$ minimale. Cette valeur minimale est à peu près indépendante de la vitesse de déformation relative axiale $\dot{\varepsilon}$ ou de la vitesse de charge axiale $\dot{\sigma}$ pour une composition de mélange injecté donnée, mais elle dépend de cette composition. Ensuite, survient un phénomène de foisonnement qui s'amplifie rapidement et entraîne la rupture générale de l'éprouvette.

Remarquons que pour ces essais $\frac{\Delta V}{V}=\frac{1-2 v}{E} \sigma_{1}$, car :

$$
\begin{aligned}
& \text { E } \varepsilon_{1}=\sigma_{1} \\
& \text { E } \varepsilon_{2}=-v \sigma_{1} \\
& \text { E } \varepsilon_{3}=-v \sigma_{1}
\end{aligned}
$$

\subsection{Evolution du module de déformabilité $E$}

Les essais ont montré que le matériau est d'autant plus raide que la vitesse de déformation relative axiale $\dot{\varepsilon}$ (ou la vitesse de charge axiale $\dot{\sigma}$ ) est grande.

Pour des valeurs de $\dot{\varepsilon}$ et $\dot{\sigma}$ données, le module de déformabilité dépend de la composition du mélange injecté.

\subsection{Evolution de la résistance maximale $R_{c}$}

La résistance maximale $\mathrm{R}_{c}$ croît régulièrement avec la vitesse de déformation $\dot{\varepsilon}$ ou avec la vitesse de charge $\dot{\sigma}$.

Elle est fortement influencée par la composition du mélange injecté. Les trois tendances générales qui ont été, soit simplement vérifiées, soit mises en évidence sont :

1) Pour une dilution constante $(\beta=c t e), R_{c}$ décroît si $\mathrm{N} \%($ ou $\alpha)$ décroît.

2) Pour une teneur en réactif constante ( $N \%$ ou $\alpha=$ cte), $\mathrm{R}_{c}$ décroît si la dilution augmente $(\beta \uparrow)$.

3) L'influence des vitesses $\dot{\varepsilon}$ ou $\dot{\sigma}$ est d'autant plus forte que $\mathrm{N} \%($ ou $\alpha)$ est plus faible. Ce dernier point est évidemment d'importance capitale si on

Fig. 9. - Compression simple avec vitesse a contrôlée. Dosage IV $_{B}$.

Fig. 10. - Influence de la vitesse de déformation contrôlée $\dot{\varepsilon}_{1}$ sur $\mathbf{R}_{c}$. Gels de composition $\mathrm{N}=30 \% \quad(\alpha=0.085)$.

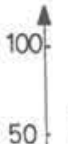

$\mathrm{R}_{\mathrm{c}}$ $10^{5} \mathrm{~Pa}$ $\mathrm{V}_{\mathrm{C}} \quad \mathrm{III}_{\mathrm{B}} \quad \mathrm{IV}_{\mathrm{B}}$
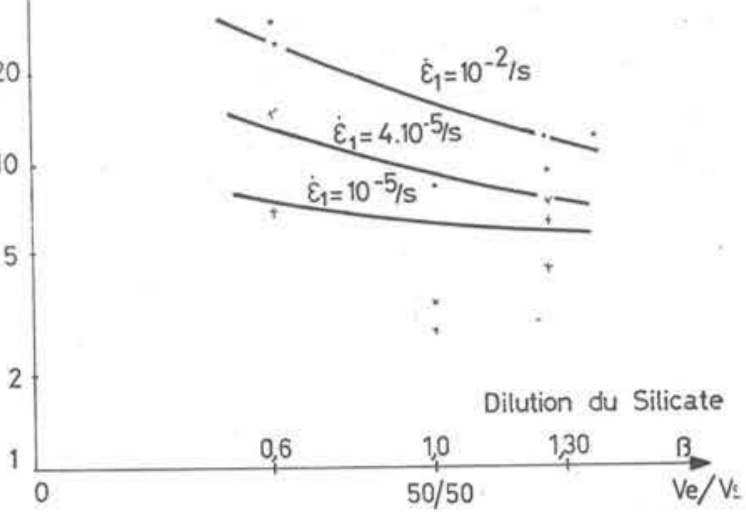

Fig. 11. - Influence de la vitesse de déformation contrôlée $\dot{\varepsilon}_{i}$ sur $\mathbf{R}_{c}$. Gels de composition $\mathrm{N}=70 \%(\alpha=0.197)$.

咅

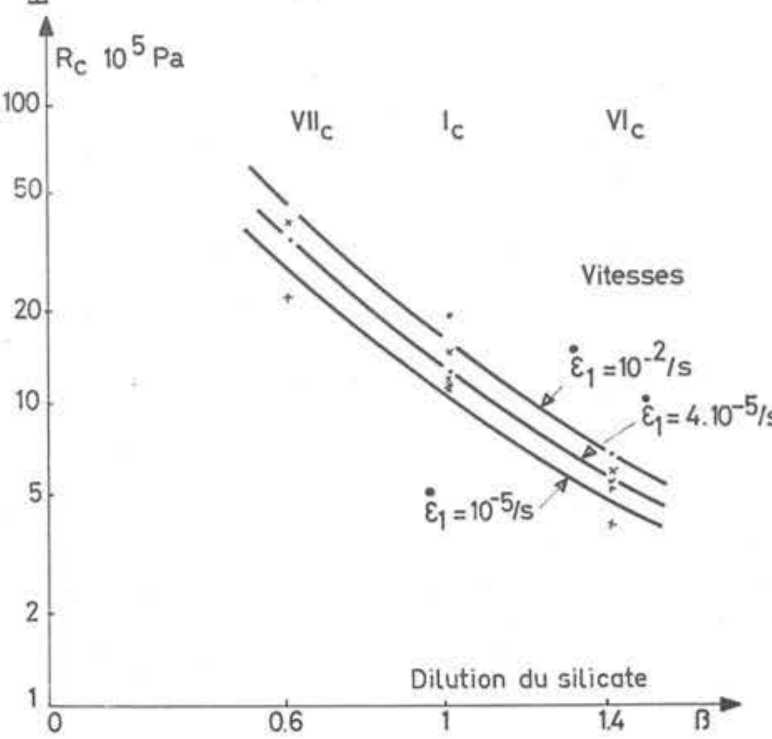


se reporte à la définition actuelle du critère de qualité d'un traitement de consolidation de terrain par injection de produits chimiques.

Les deux figures 10 et 11 illustrent bien ces faits. On peut y remarquer que, pour une dilution identique, les écarts entre les valeurs de $R_{c}$ pour les trois vitesses $\dot{\varepsilon}$ sont beaucoup plus importants pour le gel $\mathrm{N}=30 \%$ que pour le gel $\mathrm{N}=70 \%$

\subsection{Relaxation}

Les essais de relaxation ont mis en évidence la complexité du comportement rhéologique de ces matériaux.

Fig. 12. - Sable injecté avec un gel de silicate de soude. Phénomène de relaxation.
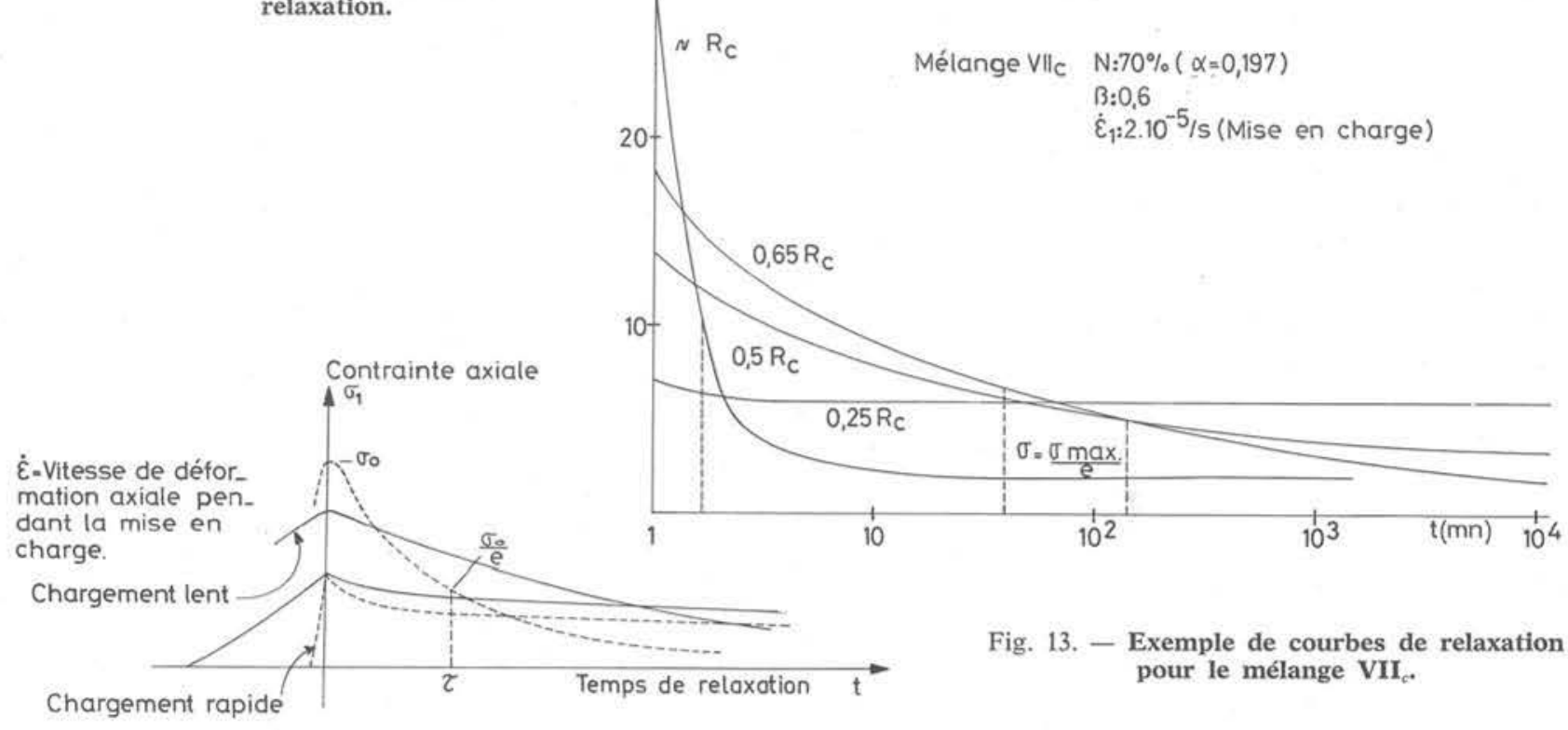

3) Ensuite se développe une phase de fluage établi, mise en évidence par un segment quasi-rectiligne dans le diagramme $\left(\varepsilon_{1}, \log t\right)$.

4) Cette phase se poursuit par une phase de fluage accéléré qui précède la rupture.

Des constatations classiques ont pu être faites : pour une charge relativement importante, il n'y a pas de fluage établi et le fluage accéléré fait suite à la déformation instantanée. Dans le cas d'une charge relativement faible, on observe un fluage asymptotique pendant des mois sans qu'apparaisse la phase accélérée.

Rappelons préalablement que l'échantillon est mis en charge jusqu'au niveau de contrainte $\sigma_{1}$ avec une vitesse de déformation relative axiale $\dot{\varepsilon}$ contrôlée.

Deux constations principales ont pu être faites (voir fig. 12 et 13$)$ :

1) La relaxation est d'autant plus importante que la contrainte maximale $\sigma_{1}$ s'approche de la charge de rupture $\mathrm{R}_{c}$.

2) $\mathrm{Si} \dot{\varepsilon}_{1}$ augmente, c'est-à-dire si l'éprouvette est chargée au niveau de contrainte $\sigma_{1}$ plus rapidement, la chute de contrainte par relaxation est plus importante. Rappelons que la contrainte de rupture $\mathrm{R}_{c}$ croît en même temps que $\dot{\varepsilon}_{1}$.

\subsection{Fluage}

Les essais de fluage sous charge constante ont donné des courbes expérimentales d'allure classique (fig. 14), c'est-à-dire :

1) Au chargement, on constate une déformation instantanée $\varepsilon$, caractéristique du comportement immédiat.

2) Juste après, se manifeste une phase de fluage primaire ou ralenti.

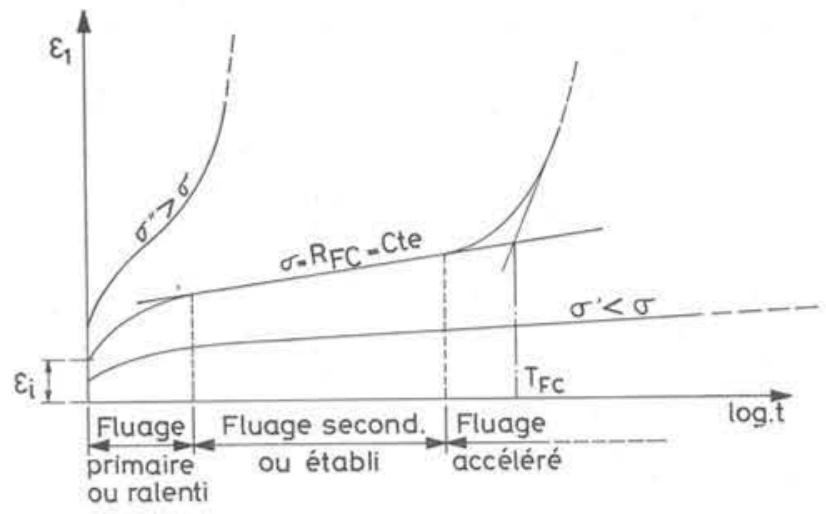

Fig. 14. - Allure des courbes de fluage sous charge statique.

Les courbes expérimentales permettent de définir une durée $\mathrm{T}_{\mathrm{FC}}$ de fluage établi, correspondant à la charge pratique $\sigma=\mathrm{R}_{\mathrm{FC}}=$ cte, appelée résistance au fluage en compression simple associée à la durée $\mathrm{T}_{\mathrm{FC}}$.

La figure 15 , reproduite à titre d'exemple, représente les courbes de fluage sous les charges statiques $\sigma=2 \mathrm{MPa} ; 1.6 \mathrm{MPa}$ et $0.8 \mathrm{MPa}$ telles qu'elles ont été obtenues pour le mélange XIV BE. 
Fig. 15. - Courbes de fluage du mélange $\mathbf{X I V}_{B E}$.

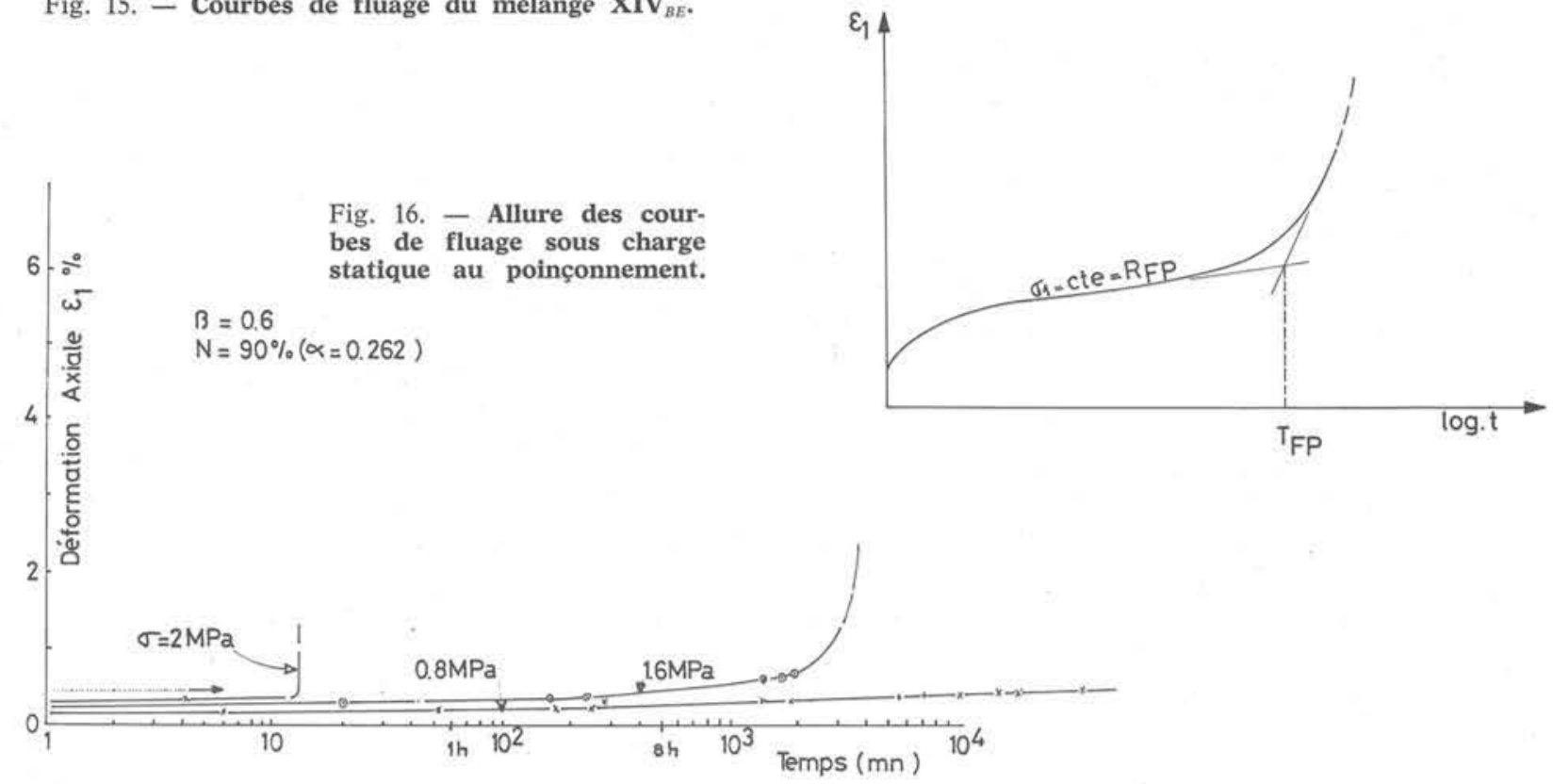

Fig, 17. - Fluage en poinçonnement du mélange $\mathbf{X}_{c}$.

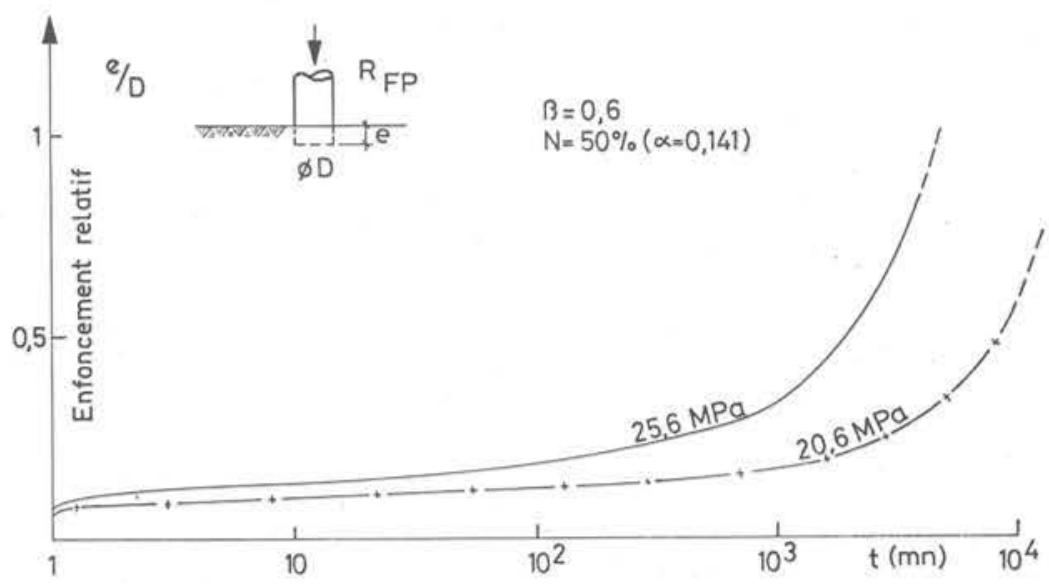

\subsection{Résistance statique sous l'effet de poinçonnement}

Les essais montrent que les courbes de l'enfoncement relatif $e / D$ en fonction du temps (fig. 17), ont la même allure que les courbes de fluage en compression simple $\left(\varepsilon_{1}, \log t\right)$ (fig. 16). Il est donc

\section{CONSTATATIONS EXPERIMENTALES SUR LES SABLES INJECTES AUX RESINES ACRYLAMIDES}

Les essais ont été en nombre plus restreint sur ce matériau car, comme il est expliqué au paragraphe 1.22 , il s'agissait d'établir une comparaison de comportement avec celui des sables injectés avec des gels de silicate.

Les essais ont été les suivants :

a) essais de compression simple avec vitesse de déformation relative axiale $\dot{\varepsilon}_{1}$ contrôlée ;

b) essais de fluage en compression simple sous charge statique :

c) essais de fluage en poinçonnement sous charge statique, avec un poinçon de $0.5 \mathrm{~cm}^{2}$ de section. possible de définir également une résistance au fluage en poinçonnement $R_{F P}$, associée à une durée de fluage établi $\mathrm{T}_{\mathrm{FP}}$

La figure 17 montre l'exemple des courbes obtenues sur le mélange $\mathrm{X}_{c}$ avec les contraintes de poinçonnement de $25.6 \mathrm{MPa}$ et $20.6 \mathrm{MPa}$.

\subsection{Evolution de la résistance maximale $R_{c}$}

De même que pour les sables injectés avec des gels de silicate, la résistance maximale $\mathrm{R}_{c}$ croît régulièrement avec la vitesse de déformation axiale $\dot{\varepsilon}$. Elle est également influencée par la composition des mélanges :

1) $R_{c}$ croît avec la teneur en extrait sec ES \%.

2) Pour une même teneur en extrait sec, $R_{c}$ croît avec la teneur en reticulant TR \% mais tend vers une asymptote pour $\mathrm{TR}=4$ à $5 \%$.

3) L'influence de la vitesse de déformation axiale $\varepsilon$ est du même ordre de grandeur pour des teneurs en extrait sec variables.

4) L'influence de $\dot{\varepsilon}$ est d'autant plus sensible que la teneur en reticulant TR est élevée. 


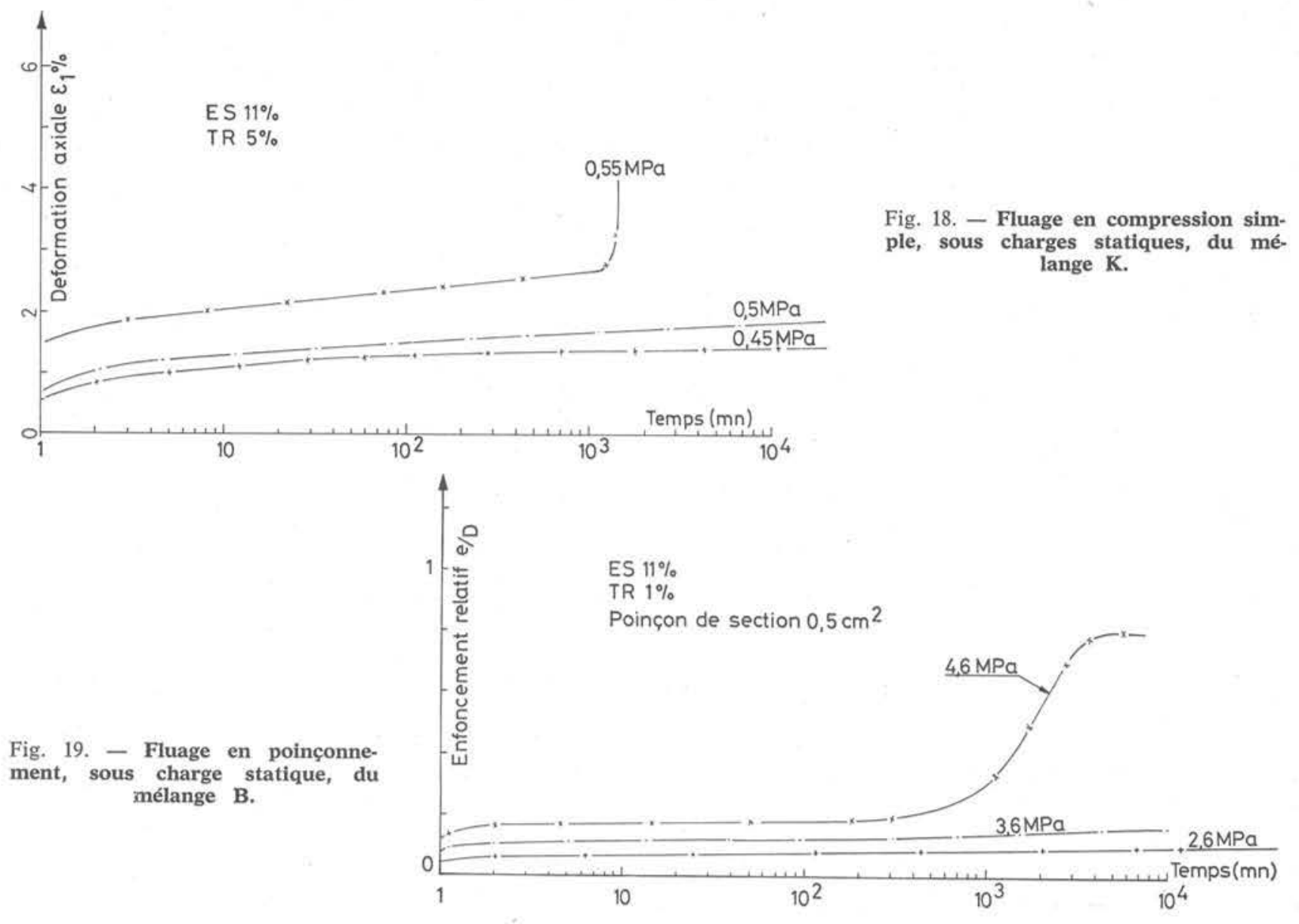

\subsection{Fluage}

Les courbes de fluage ont été déterminées uniquement pour les mélanges contenant $11 \%$ d'extrait sec car il s'agit du dosage utilisé le plus couramment.

Le comportement au fluage montre que la résistance $R_{F C}$ est plus faible que $R_{c}$ mais elle varie très peu avec la durée de fluage établi.

La figure 18 montre, à titre d'exemple, les résultats obtenus sur le mélange $\mathrm{K}(\mathrm{ES}=11 \%, \mathrm{R}=5 \%)$ pour des charges statiques correspondant à $0.45 \mathrm{MPa}$; $0.5 \mathrm{MPa}$ et $0.55 \mathrm{MPa}$.

\subsection{Poinçonnement}

Les essais de fluage en poinçonnement ont été réalisés également sur les mélanges $\mathrm{ES}=11 \%$. La résistance $R_{F P}$ sous charge constante varie de façon analogue à la charge de fluage $R_{\mathrm{FC}}$.

La figure 19 montre l'allure des courbes obtenues sur le mélange B pour des charges statiques correspondant à $2.6 \mathrm{MPa} ; 3.6 \mathrm{MPa}$ et $4.6 \mathrm{MPa}$.

\section{INTERPRETATION DES RESULTATS OBTENUS SUR LES SABLES INJECTES AUX GELS DE SILICATE}

\subsection{Résistance à la compression simple}

Les valeurs quantitatives obtenues à partir des essais de compression simple, qu'il s'agisse des essais avec $\dot{\varepsilon}=$ cte ou $\dot{\sigma}=$ cte, présentent une certaine dispersion qui est propre à ce genre d'expérimentation où le nombre des opérations diverses est très élevé.

Les résultats peuvent être représentés graphiquement de façon simple sous la forme de courbes de régression linéaire par la méthode des moindres carrés, calculées en coordonnées normales et semi-logarithmiques. Ces courbes représentent les lois empiriques de la forme :

ou

$$
\mathrm{R}_{c}=\mathrm{R}_{o}+\rho \dot{\varepsilon} \text { (ou } \dot{\sigma} \text { ) }
$$

$$
\mathrm{R}_{c}=\mathrm{R}_{o}^{\prime}+\rho^{\prime} \log \dot{\varepsilon}(\text { ou } \dot{\sigma})
$$

La relation (2) ne présente pas de signification théorique et elle n'est qu'un ajustement expérimental à une loi logarithmique.

La relation (1) peut, par contre, être représentée par un modèle rhéologique de Bingham (fig. 20). En effet, lorsqu'on comprime un modèle de Bingham, avec une vitesse de déformation $\dot{\varepsilon}=$ cte, la contrainte obtenue, à partir de l'instant $t_{o}=\frac{\mathrm{S}}{\mathrm{G} \dot{\varepsilon}}$ où le patin commence à glisser, est :

où

$$
\sigma=\mathrm{S}+\eta \dot{\varepsilon}\left\{1-\exp \left(\frac{t-t_{o}}{\tau}\right)\right\}
$$

$\eta$, est la viscosité de l'amortisseur ; 


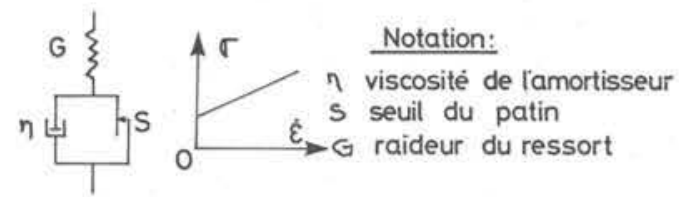

Fig. 20. - Modèle de Bingham.

S, le seuil du patin;

$\mathrm{G}$, la raideur du ressort;

$\tau=\frac{\eta}{\mathrm{G}}$ le temps de retard.

Le comportement réel du matériau étudié pourrait être représenté par la superposition de plusieurs modèles élémentaires de Bingham.

Les figures 21 et 22 montrent les courbes de régression linéaire obtenues pour les essais avec $\dot{\sigma}=$ cte et $\dot{\varepsilon}=$ cte pour le mélange $\mathrm{V}_{c}$.

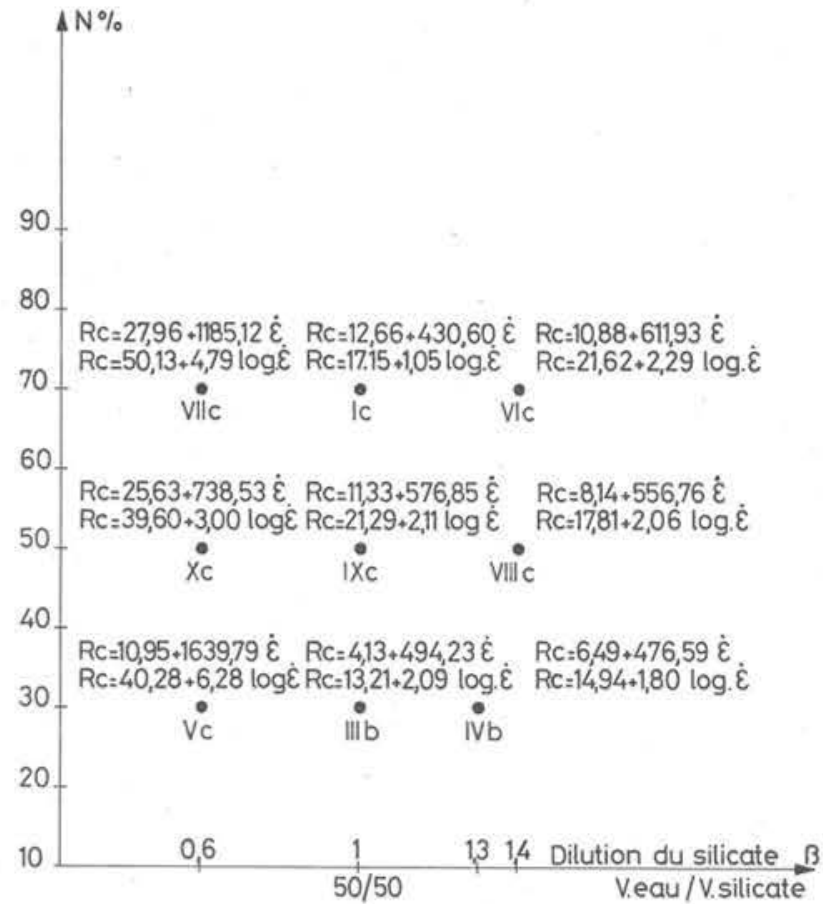

Fig. 23, - Gels de silicate. Equations des courbes de régression linéaire (avec $\dot{\varepsilon}=$ cte),

L'ensemble des relations empiriques expérimentales, exprimées sous les formes (1) et (2), obtenues pour les essais réalisés avec $\dot{\varepsilon}=$ cte est reporté sur la figure 23 .

Ces relations, définies uniquement dans le cadre des valeurs expérimentales, ne permettent pas l'extrapolation et ces deux tentatives montrent bien la difficulté d'approcher les résultats physiques obtenus vers des vitesses de déformation $\dot{\varepsilon}$ ou des vitesses de mise en charge $\dot{\sigma}$ très faibles sinon nulles.

Les coefficients numériques de ces relations permettent cependant de constater que, dans le domaine des vitesses de déformations axiales $\dot{\varepsilon}$ utilisées, la résistance à la rupture en compression simple $\mathbf{R}_{c}$ est affectée d'une façon générale, comme suit :

1) La sensibilité aux variations de la vitesse de déformation $\dot{\varepsilon}$ diminue avec une augmentation de la dilution du silicate $(\beta \uparrow)$.

2) La sensibilité aux variations de la vitesse $\dot{\varepsilon}$ est plus marquée pour des taux de neutralisation bas ( $\mathrm{N} \%$ ou $\alpha \downarrow)$.

Les valeurs expérimentales réelles de $\mathrm{R}_{c}$ obtenues en fonction des diverses valeurs de $\dot{\varepsilon}$ sont consignées, plus loin, dans le tableau de la figure 27 où elles ont été groupées avec les résultats de la résistance à la rupture sous charge statique pour visualiser l'influence du facteur temps de façon plus significative. 


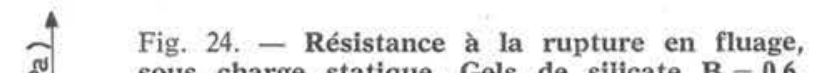
sous charge statique. Gels de silicate $B=0,6$.
Les valeurs extrêmes sont données par les essais de fluage car une fois les mises sous contrainte réalisées, $\dot{\sigma}=0$.

Les courbes expérimentales ont montré que la résistance au fluage sous charge statique $\mathrm{R}_{\mathrm{FC}}$ était associée à une durée $\mathrm{T}_{\mathrm{FC}}$.
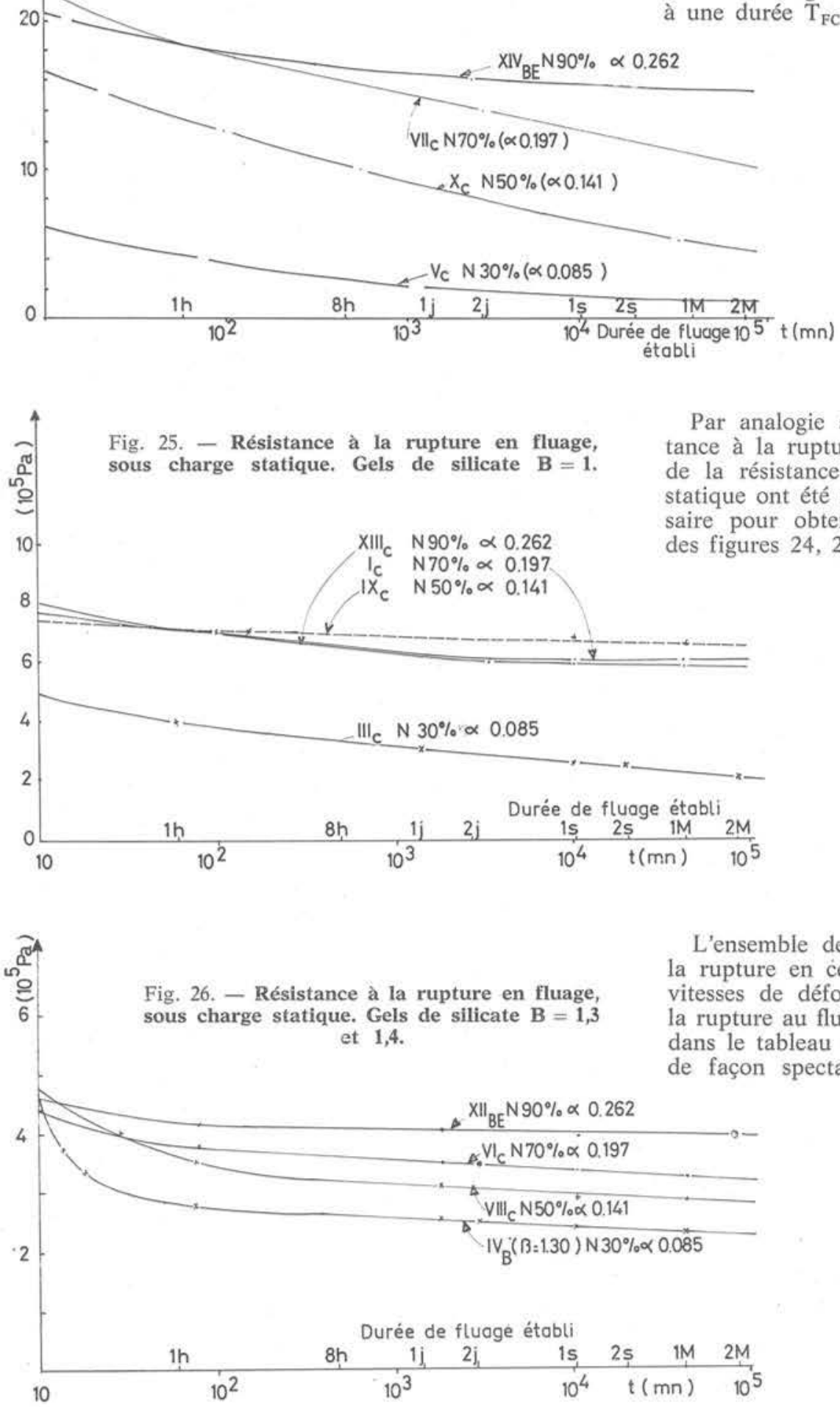

\subsection{Fluage}

Les valeurs de la résistance mécanique intéressantes pour la pratique sont celles que l'on peut utiliser, avec sécurité, dans des calculs de forces portantes, de butées, etc. Ces valeurs sont obtenues dans les conditions où $\dot{\varepsilon}$ ou $\dot{\sigma}$ tendent vers zéro. sur la résistance à la compression du sable injecté avec des gels de silicate. La colonne $R_{1} / R_{4}$ correspond au rapport des résistances à la compression simple obtenues avec les vitesses de déformation axiale $\dot{\varepsilon}=4 \cdot 10^{-3} / \mathrm{s}$ et $4 \cdot 10^{-6} / \mathrm{s}$. 


\begin{tabular}{|c|c|c|c|c|c|c|c|c|c|c|c|c|}
\hline \multirow{2}{*}{ 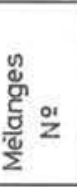 } & \multirow{2}{*}{ 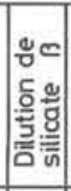 } & \multirow{2}{*}{ 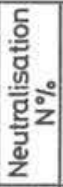 } & \multirow{2}{*}{ 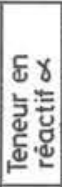 } & \multicolumn{5}{|c|}{$\begin{array}{l}\text { Résistance à la comp. simple } \\
\text { ( } 10^{5} \mathrm{~Pa} \text { ) à différentes vitesses } \\
\text { de déformation है } \\
1\end{array}$} & \multicolumn{4}{|c|}{$\begin{array}{l}\text { Résistance sous charge cons. } \\
\left.\text { tante ( } 10^{5} \mathrm{~Pa}\right) \sigma^{\circ}=0 \text { (fluage) } \\
\text { apres : }\end{array}$} \\
\hline & & & & $4.10^{-3} / \mathrm{s}$ & $4.10^{-4} / \mathrm{s}$ & $4.10^{-5} / \mathrm{s}$ & $4.10^{-6} / \mathrm{s}$ & $\mathrm{R} 1 / \mathrm{R}_{4}$ & $1 \mathrm{~h}$. & $1 \mathrm{j}$. & $1 \mathrm{~s}$. & $1 \mathrm{~m}$ \\
\hline$v_{C}$ & 0.6 & 30 & 0.085 & 25.2 & 19.0 & 12.7 & 6.4 & 3.9 & 4.1 & 2.0 & 1.4 & 1.2 \\
\hline$x_{C}$ & 0.6 & 50 & 0.141 & 32.5 & 29.5 & 26.5 & 23.5 & 1.38 & 134 & 8.8 & 6.5 & 5.1 \\
\hline $\mathrm{VII}_{\mathrm{C}}$ & 0.6 & 70 & 0.197 & 39.0 & 34.0 & 29.2 & 24.5 & 1.58 & 18.4 & 14.8 & 12.6 & 11.0 \\
\hline $\mathrm{XIV}_{B E}$ & 0.6 & 90 & 0.262 & & & & & & 18.4 & 16.3 & 15.6 & 15.2 \\
\hline $\mathrm{iil}_{\mathrm{B}}$ & 1.0 & 30 & 0.085 & 8.2 & 6.2 & 4.1 & 2.0 & 4.1 & 3.9 & 3.0 & 2.6 & 2.2 \\
\hline${ }^{I} x_{C}$ & 1.0 & 50 & 0.141 & 16.0 & 14.0 & 12.0 & 10.0 & 1.6 & 7.2 & 6.8 & 6.7 & 6.5 \\
\hline IC & 1.0 & 70 & 0.204 & 14.8 & 13.6 & 12.6 & 11.5 & 1.27 & 7.2 & 6.2 & 6.0 & 6.0 \\
\hline$x \|_{B E}$ & 1.0 & 90 & 0262 & & & & & & 7.2 & 6.3 & 5.9 & 5.8 \\
\hline $\mathrm{IV}_{\mathrm{B}}$ & 1.3 & 30 & 0.084 & 107 & 9.0 & 7.1 & 5.3 & 2.2 & 2.8 & 2.5 & 2.4 & 2.3 \\
\hline VIIIIC & 1.3 & 50 & 0.141 & 11.8 & 9.7 & 8.6 & 7,5 & 1.6 & 3.7 & 3.1 & 3.0 & 2.3 \\
\hline $\mathrm{VI}_{\mathrm{C}}$ & 1.4 & 70 & 0.197 & 8.6 & 7.3 & 6.6 & 5.9 & 1.5 & 3.8 & 3.5 & 3.6 & 3.2 \\
\hline$X \|_{B E}$ & 1.4 & 90 & 0.262 & & & & & & 4.2 & 4.0 & 4.0 & 4.0 \\
\hline
\end{tabular}

Fig. 27. - Gels de silicate. Tableau récapitulatif des résistances à la compression simple $R_{c}$ en fonction de la vitesse $\dot{\varepsilon}$ et des résistances à la rupture au fluage en fonction du temps.

Avec les dimensions des échantillons utilisés, ces vitesses correspondent à des déplacements de plateaux de presse d'écrasement de $2 \mathrm{~cm} / \mathrm{mn}$ et $0.02 \mathrm{~cm} / \mathrm{mn}$. Ces vitesses sont les valeurs extrêmes rencontrées le plus fréquemment et les valeurs du rapport de la colonne $R_{1} / R_{4}$ illustrent bien la différence de signification que peut avoir un résultat de résistance à la compression simple lorsque la vitesse de déformation axiale n'est pas précisée.

\subsection{Poinconnement}

Les courbes expérimentales ont montré qu'il était possible de définir une résistance au fluage par poinçonnement $\mathrm{R}_{\mathrm{FP}}$ associée à une durée de fluage établi $T_{\mathrm{FP}}$. Il était intéressant de comparer les résistances $\mathrm{R}_{\mathrm{FP}}$ qui correspondent à une distribution de contraintes non homogène, aux résistance $\mathrm{R}_{\mathrm{FC}}$ obtenues pendant les essais de fluage en compression simple (état de contrainte uniforme). Cette comparaison s'est faite au niveau des résistances $R_{F C}$ et $R_{F P}$, estimées sur les graphiques du type de la figure 17 , pour des durées

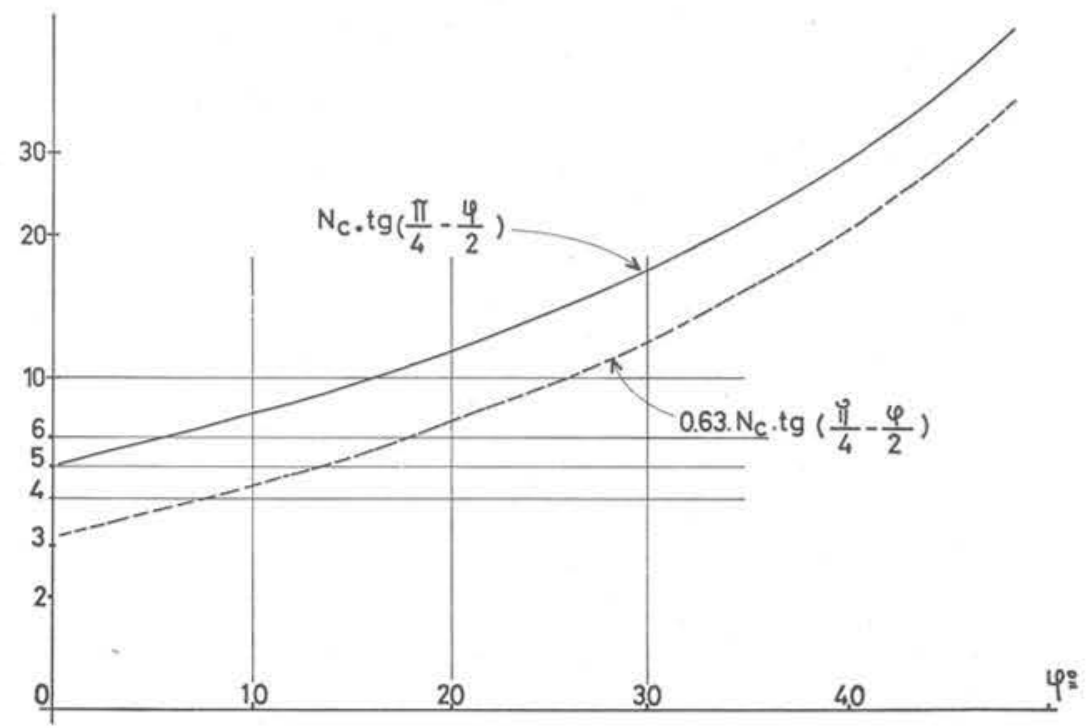

$T_{F C}$ et $T_{F P}$ identiques. Elle est exprimée sous forme du rapport :

$$
\frac{\text { résistance au fluage en poinçonnement } R_{F P}}{\text { résistance au fluage en compression } R_{F C}}
$$

En appliquant la formule générale de portance et en négligeant le terme de surface (car la section du poinçon est petite : $0.5 \mathrm{~cm}^{2}$ ) et le terme de profondeur (fondation superficielle), la résistance au fluage en poinçonnement peut s'écrire :

$$
\mathrm{R}_{\mathrm{FP}}=1.25 \mathrm{C} \mathrm{N}_{c}+0.7 \frac{\gamma \mathrm{B}}{2} \mathrm{~N}_{\gamma}+\gamma \mathrm{D} \mathrm{N}_{q} \rightarrow 1.25 \mathrm{C} \mathrm{N}_{c}
$$

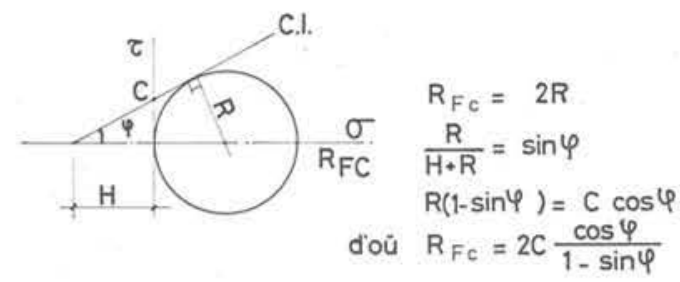

Fig. 28. - Diagramme de Mohr. 
Fig. 30. - Valeurs expérimentales du rapport $\frac{\mathbf{R}_{F P}}{\mathbf{F}_{F C}}$.

\begin{tabular}{|c|c|c|c|c|c|}
\hline \multicolumn{6}{|c|}{$N 50 \% \quad \alpha=0.141$} \\
\hline $\mathrm{VIII}_{\mathrm{C}}$ & $B=1,4$ & $\mathrm{~T}=1200 \mathrm{mn}$ & $R_{E P}=25.10^{5} \mathrm{~Pa}$ & $R_{E}=3,1.10^{5} \mathrm{~Pa}$ & $\frac{R_{F P}}{R}=8,06$ \\
\hline $\mathrm{Ix}_{\mathrm{C}}$ & 1 & $300 \mathrm{mn}$ & $25.10^{5} \mathrm{~Pa}$ & $\quad 7.10^{5} \mathrm{~Pa}$ & ${ }^{\mathrm{R} F C} \quad 3,57$ \\
\hline$x_{C}$ & 0.6 & $3000 \mathrm{mn}$ & $256.10^{5} \mathrm{~Pa}$ & $8.10^{5} \mathrm{~Pa}$ & 32 \\
\hline${ }^{x_{C}}$ & 0,6 & $10^{4} \mathrm{mn}$ & $206.10^{5} \mathrm{~Pa}$ & $6,5 \cdot 10^{5} \mathrm{~Pa}$ & 31.69 \\
\hline \multicolumn{6}{|c|}{$\mathrm{N} 70 \% \quad \alpha=0.197$} \\
\hline $\mathrm{VI}_{\mathrm{C}}$ & 1,4 & $10^{5} \mathrm{mn}$ & $25.10^{5} \mathrm{~Pa}$ & $3,3.10^{5} \mathrm{~Pa}$ & 7.58 \\
\hline \multicolumn{6}{|c|}{$\mathrm{N} 90 \% \quad \alpha=0.262$} \\
\hline & 1.4 & $10^{4} \mathrm{mn}$ & $45.10^{5} \mathrm{~Pa}$ & $4.10^{5} \mathrm{~Pa}$ & 11.25 \\
\hline XIV & 0.6 & $10^{4} \mathrm{mn}$ & $300.10^{5} \mathrm{~Pa}$ & $15.5 .10^{5} \mathrm{P}$ & 19.35 \\
\hline
\end{tabular}

D'autre part, le diagramme de Mohr montre que la résistance en compression simple (fig. 28) à :

$$
\mathrm{R}_{\mathrm{FC}}=2 \mathrm{R}
$$

où $\mathrm{R}$ est le rayon du cercle de Mohr :

$$
\mathrm{R}=(\mathrm{H}+\mathrm{R}) \sin \varphi
$$

d'autre part, $\mathrm{H}=\mathrm{C} \operatorname{cotg} \varphi$

d'où $\mathrm{R}(1-\sin \varphi)=\mathrm{C} \cos \varphi$

$$
\begin{aligned}
& \text { et } \mathrm{R}_{\mathrm{F}_{\mathrm{C}}}=2 \mathrm{R}=\frac{2 \mathrm{C} \cos \varphi}{1-\sin \varphi} \\
& \frac{\mathrm{R}_{\mathrm{FD}}}{\mathrm{R}_{\mathrm{F}_{\mathrm{C}}}}=\frac{1.25 \mathrm{C} \mathrm{N}(1-\sin \varphi)}{2 \mathrm{C} \cos \varphi} \approx 0.63 \mathrm{~N}_{c} \operatorname{tg}\left(\frac{\pi}{4}-\frac{\varphi}{2}\right)
\end{aligned}
$$

Ce rapport devrait permettre d'évaluer la mobilisation de l'angle de frottement interne; des écarts restent possibles dans la mesure où la rupture sous un poinçon est progressive.

Les valeurs théoriques de ce rapport sont reportées sur le graphique de la figure 29 .

Les valeurs expérimentales obtenues sont consignées dans le tableau de la figure 30 . On peut y remarquer que la valeur de ce rapport n'est pas constante et qu'elle diminue lorsque la dilution du Silicate augmente $(\beta \uparrow)$. Une telle expérimentation demande à être développée car il semble que la valeur du rapport soit une fonction plus complexe de $\varphi$.

\section{INTERPRETATION DES RESULTATS OBTENUS SUR LES SABLES INJECTES AUX RESINES ACRYLAMIDES}

\subsection{Résistance à la compression simple}

De même que pour l'étude conduite avec les gels de silicate, les valeurs expérimentales obtenues à partir des essais de compression simple, avec $\dot{\varepsilon}=$ cte ou $\dot{\sigma}=$ cte, peuvent être représentées graphiquement sous la forme de courbes de régression linéaire correspondant aux équations (1) et (2) du paragraphe 5.1. Les valeurs numériques provenant des essais avec $\dot{\varepsilon}=$ cte conduisent aux équations (1) et (2) regroupées dans le tableau de la figure 31 .

Mis à part les valeurs concernant le mélange $H$, l'ensemble des résultats est très cohérent et conduit aux constatations suivantes :

1) Les pentes des droites sont moins accentuées que celles correspondant aux gels de silicate. Les résistances à la compression simple sont donc moins sensibles à l'influence de la valeur de la vitesse de déformation axiale $\dot{\varepsilon}$.

2) La sensibilité à la vitesse $\dot{\varepsilon}$ reste du même ordre de grandeur pour des teneurs en extrait sec variables.

3) Pour une même teneur en extrait sec, la sensibilité à la vitesse $\dot{\varepsilon}$ augmente avec la teneur en réticulant ; ce fait se vérifie bien pour chaque pourcentage d'extrait sec.

4) Pour une même vitesse de déformation axiale $\dot{\varepsilon}=10^{-4} / \mathrm{s}$, les valeurs réelles de la résistance $R_{c}$ sont reportées sur les figures 32 et 33 . On y vérifie que :

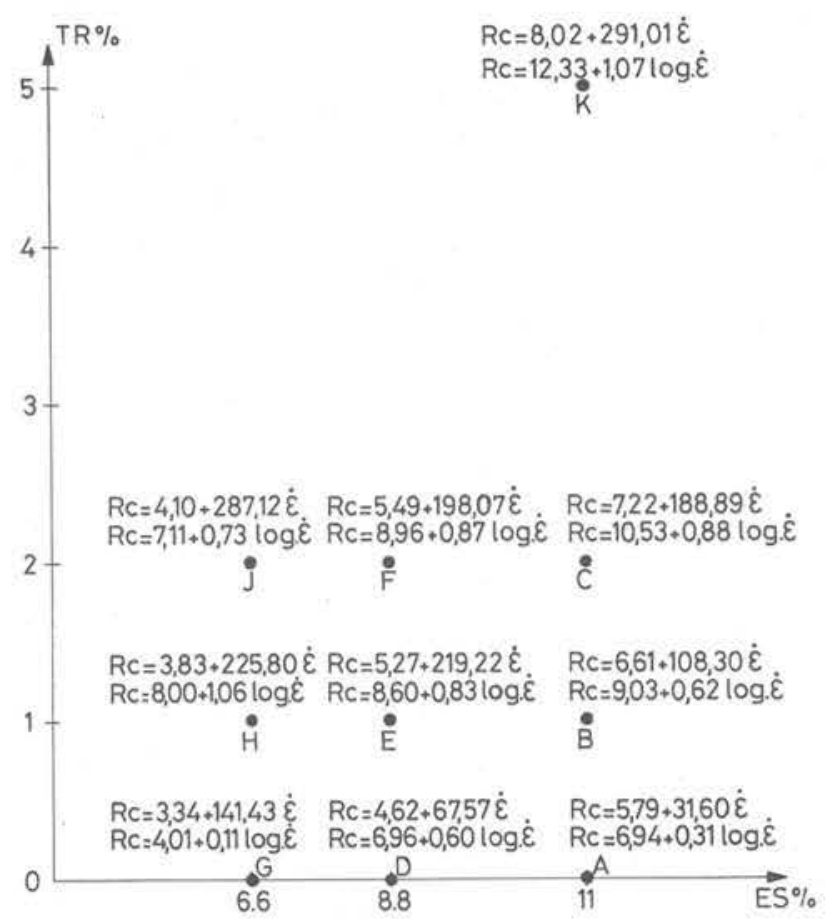

Fig. 31. - Résines acrylamides. Equations des courbes de régression linéaire (avec $\dot{\varepsilon}=$ cte). 

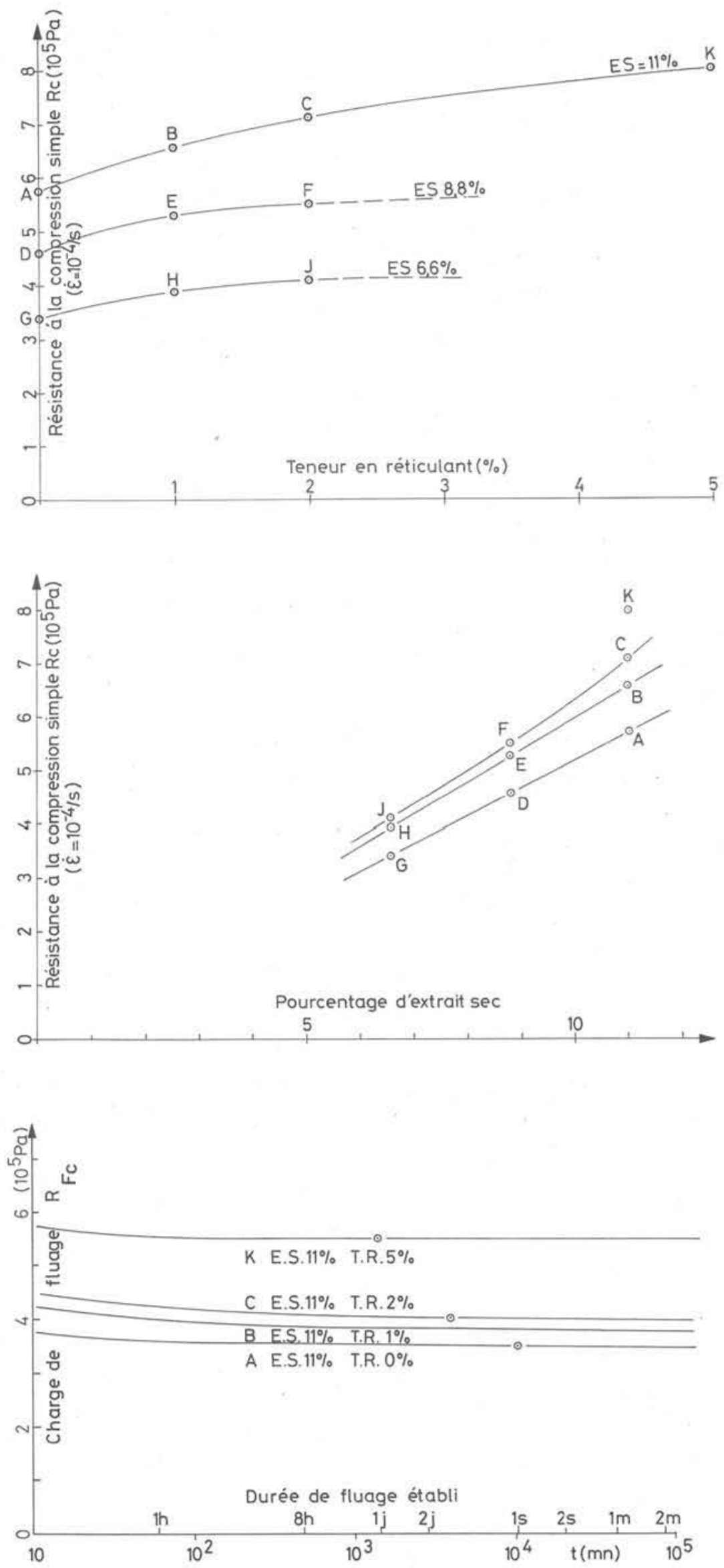

Fig. 32. - Résines acrylamides. Influence de la teneur en réticulant sur $\mathbf{R}_{c}$.

a) la résistance à la compression simple $\mathrm{R}_{c}$ croît proportionnellement à la teneur en extrait sec ES \%;

b) la résistance $R_{c}$ augmente avec la teneur en réticulant TR \% mais tend rapidement vers une asymptote à partir de $\mathrm{R}=4$ à $5 \%$.

Cette influence est d'autant plus significative que la teneur en extrait sec est élevée.

De même que pour l'étude des sables injectés aux gels de silicate, les valeurs expérimentales de $R_{c}$ obtenues pour des valeurs différentes de $\dot{\varepsilon}$ sont regroupées plus loin, dans le tableau de la figure 35 , avec les résultats de la résistance au fluage sous charge statique.

\subsection{Fluage sous charge statique}

Les essais de fluage en compression à long terme, sous charge statique, ont été conduits sur les mélanges contenant un pourcentage d'extrait $\sec \mathrm{ES}=11 \%$.

L'évaluation de la résistance à la rupture en fonction du temps pour atteindre cette rupture (fig. 34) conduit à des courbes pratiquement linéaires de pente très peu marquée. La charge de fluage $R_{F C}$ varie donc

Fig. 33. - Résines acrylamides. Influence de la teneur en extrait sec sur $\mathbf{R}_{e}$.

très peu avec la durée de fluage établi $\mathrm{T}_{\mathrm{FC}}$.

L'ensemble des résultats portant sur la résistance $R_{c}$ en fonction de diverses valeurs de $\dot{\varepsilon}$ et sur la résistance $\mathrm{R}_{\mathrm{FC}}$ est regroupé dans le ta-

Fig. 34. - Résines acrylamides. Résistance à la rupture en fluage, sous charge statique.

bleau de la figure 35 . On y remarque bien :

1) le niveau de résistance relativement bas obtenu avec les divers dosages. Ce fait ne doit pas être éliminatoire car dans certains types de travaux, ce niveau peut être suffisant.

2) La bonne stabilité dans le temps des résistances obtenues. 
Fig. 35. - Résines acrylamides. Tableau récapitulatif :

- des résistances à la compression simple $\mathbf{R}_{c}$ en fonction de la vitesse $\varepsilon$;

- des résistances à la rupture au fluage en fonction du temps.

\begin{tabular}{|c|c|c|c|c|c|c|c|c|c|c|c|}
\hline \multirow[t]{2}{*}{ Formule } & \multirow[t]{2}{*}{ E.S. } & \multirow[t]{2}{*}{ T.R. } & \multicolumn{5}{|c|}{$\begin{array}{l}\text { Résistance à la compression } \\
\text { simple sous différentes vites- } \\
\text { ses de déformation }\left(10^{5} \mathrm{~Pa}\right) \\
1\end{array}$} & \multicolumn{4}{|c|}{$\begin{array}{l}\text { Résistance sous charge cons- } \\
\text { tante en fonction de la durée } \\
\left.\text { de fluage établi ( } 10^{5} \mathrm{~Pa}\right)\end{array}$} \\
\hline & & & $4.10^{-3} \%$ & $4.10^{-4} / \mathrm{s}$ & $4.10^{-5} / \mathrm{s}$ & $4.10^{-6} / \mathrm{s}$ & & $1 \mathrm{~h}$. & $1 \mathrm{j}$. & $1 \mathrm{~s}$. & $1 \mathrm{~m}$. \\
\hline A & $11 \%$ & $0 \%$ & 6,2 & 5,9 & 5,6 & 5,3 & 1,17 & 3,6 & 3,5 & 3,5 & 3,5 \\
\hline B & - & $1 \%$ & 7,5 & 7,0 & 6.4 & 5,7 & 1,31 & 4,0 & 3,9 & 3,8 & 3,8 \\
\hline C & - & $2 \%$ & 8,4 & 7,6 & 5,7 & 5,8 & 1,44 & 4,2 & 4,1 & 4,0 & 4,0 \\
\hline K & - & $5 \%$ & 9,7 & 8,7 & 7,7 & 6,6 & 1,46 & 5,5 & 5,5 & 5,5 & 5,5 \\
\hline D & $8,8 \%$ & $0 \%$ & 5,5 & 4,9 & 4,3 & 3,7 & 1,48 & & & & \\
\hline$E$ & - & $1 \%$ & 6,6 & 5,8 & 5,0 & 4,2 & 1,57 & & & & \\
\hline $\mathrm{F}$ & - & $2 \%$ & 6,8 & 6,0 & 5,1 & 4,3 & 1,58 & & & & \\
\hline G & $6,6 \%$ & $0 \%$ & 3,8 & 3,6 & 3,5 & 3,4 & 1,11 & & & & \\
\hline H & - & $1 \%$ & 5,5 & 4,4 & 3,3 & 2,2 & & & & & \\
\hline J & - & $2 \%$ & 5,4 & 4,7 & 3,9 & 3,2 & 1,68 & & & & \\
\hline
\end{tabular}

\subsection{Poinçonnement}

Les résultats obtenus par des essais de fluage sous charge statique au poinçonnement peuvent être résumés à l'aide des valeurs suivantes des rapports $\frac{R_{F P}}{R_{F C}}$

Formule $\mathrm{B}(\mathrm{ES}=11 \% \quad \mathrm{TR}=1 \%): \frac{\mathrm{R}_{\mathrm{FP}}}{\mathrm{R}_{\mathrm{FC}}}=12.11$

$$
\begin{array}{ll}
\text { Formule C }(\mathrm{ES}=11 \% & \mathrm{TR}=2 \%): \frac{\mathrm{R}_{\mathrm{FP}}}{\mathrm{R}_{\mathrm{FC}}}=8.57 \\
\text { Formule } \mathrm{K}(\mathrm{ES}=11 \% & \mathrm{TR}=5 \%): \frac{\mathrm{R}_{\mathrm{FP}}}{\mathrm{R}_{\mathrm{FC}}}=8.36
\end{array}
$$

Les valeurs de ces rapports sont relativement voisines et elles montrent une assez bonne similitude de comportement entre le poinçonnement et le fluage en compression simple sous charge statique.

\section{CONCLUSION}

En multipliant les modes de sollicitation et en mettant l'accent sur l'influence du facteur temps dans la détermination de la résistance à la compression simple d'un sable consolidé par injection de gels de silicate ou de résines acrylamides, cette étude montre la complexité du comportement mécanique de tels matériaux. Si certains essais tels que les tests de fluage au poinçonnement contribuent à améliorer les connaissances sur ce comportement, d'autres essais, tels que le fluage en compression simple poursuivis par les essais avec vitesse de déformation axiale $\dot{\varepsilon}$ contrôlée, sont déterminants et apportent un éclairage nouveau au problème de la consolidation des sols par injection de produits chimiques.

Pour le spécialiste en injection chargé de déterminer un choix de produits, cette étude met en évidence l'influence des différents paramètres de composition sur le comportement mécanique du matériau traité.

Pour améliorer et rendre son choix plus sûr, le praticien devra prendre en compte le facteur temps et considérer comme fondamental le caractère provisoire ou définitif du traitement de consolidation qu'il aura à réaliser.

Dans le cas d'un traitement définitif, (dans la mesure ou ce terme peut s'appliquer dans le domaine de la construction), c'est bien la résistance fondamentale au fluage qu'il faut prendre en compte associée au «temps de fluage établi », notions définies dans la présente étude. Cette résistance pratique à long terme sera relativement modeste et pour l'obtenir il faudra faire appel à des mélanges d'un prix de revient plus élevé (taux de neutralisation voisin de $100 \%$ pour les gels de silicate, pourcentage d'extrait sec important et teneur en reticulant de l'ordre de $5 \%$ pour les résines acrylamides).

Dans le cas d'un traitement provisoire le facteur temps est tout aussi imporfant. Le spécialiste en injection pourra choisir le mélange le plus économique compatible avec la durée de sollicitation du terrain consolidé. Cette durée est liée le plus souvent à la méthode de travail de l'entreprise générale chargée des travaux de terrassement et de génie civil. Ces facteurs devront donc également être pris en compte dans la définition du type de gel à mettre en œuvre pour la consolidation recherchée.

Pour le projeteur, qu'il s'agisse aussi bien de la phase de détermination technique d'un traitement que de son aspect contractuel, il semble désormais évident que le critère de qualité, défini comme le résultat d'une résistance à la compression simple, est insuffisant. Toute valeur annoncée doit être complétée par la définition du mode et de la vitesse de sollicitation, et il est clair qu'une seule valeur associée à une seule vitesse de sollicitation, est insuffisante.

Il ressort que pour certains types de travaux, où le mode de sollicitation du terrain traité est proche de celui de la compression simple (reprises en sous-œuvre notamment), les résultats de cette étude peuvent être transposés directement. Il ressort également que pour d'autre types de consolidation, où le mode de travail du terrain traité n'est plus semblable à la compression simple, la transformation est délicate, et peut être les calculs devraient-ils être faits à partir des méthodes 
de la mécanique des sols plutôt qu'avec celles de la résistance des matériaux classique.

De nombreux travaux de consolidation des sols ont été réalisés par injections de produits chimiques et d'autres le seront. Le contenu de cette étude devrait contribuer à l'épanouissement de cette technique car, s'il introduit quelques complexités supplémentaires aussi bien pour les projeteurs et maîtres d'œuvres que pour les spécialistes en injection, il va dans le sens d'une amélioration de la fiabilité et de la sécurité qui peuvent être apportés aux traitements de consolidation des sols par injection.

\section{BIBLIOGRAPHIE}

[1] CARON (C.). - « Etude physico-chimique des gels de silice ". Annales de l'ITBTP, série EM/81 (mars-avril 1965).

[2] KUTZNER (Ch.). - « Uber die mechanischen Eigenschaften der mit Silikatgelen injizierten Erdstoffe ». Die Bautechnik. H. 3, S. 86-97.

[3] PERSOZ (B.). - " La rhéologie " $n^{\circ}$ 3, Monographies du CAST. Masson et Cie (1969).

[4] RICHARD (C.). - - Contribution à l'étude des propriétés mécaniques d'un sable renforcé par des gels de silice. Application d'une méthode ultrasonore ». Mémoire CNAM (1975).

[5] WARNER (J.). - « Strength properties of chemically solidified soils ». Journal of the Soils Mechanics and Foundations Division. ASCE. Vol. 98, $\mathrm{n}^{\circ}$ SM. 11 (novembre 1972). 


\section{DISCUSSION}

M. le PRESIDENT, - Je tiens à féliciter $M$. Luong et toute l'équipe pour la qualité du travail qu'ils ont mené et aussi pour les résultats qu'ils ont trouvés, qui sont très intéressants.

Je suis sûr que tous les spécialistes qui sont dans la salle ne manqueront pas d'avoir des réactions. Je leur livre la parole.

M. DUFFAUT. - Vous avez vérifié que l'âge auquel vous prenez l'échantillon ne change rien à ses propriétés. Autrement dit, vous partez de produits qui sont stabilisés en âge. C'est la vitesse de charge qui compte, ce n'est pas du tout l'âge comme dans du béton. C'est clair?

M. LUONG. - C'est clair, c'est bien cela.

M. DUFFAUT. - Vous avez opéré uniquement en compression simple, sans confinement, tandis que dans le terrain, et notamment autour de souterrains, on a des chances pour que la plus grande partie des échantillons soit confinée, c'est-à-dire, qu'on n'ait pas de possibilité de changer le volume, d'augmenter le volume en tout cas. Est-ce que ca changerait quelque chose?

M. LUONG. - Vu la complexité du problème, nous avons commencé par des essais du type le plus simple possible. Donc on a étudié la résistance à la compression simple et on n'a étudié que le paramètre qui nous a semblé le plus important. Dans une phase ultérieure, il est possible qu'on étudie les sols en fonction d'autres paramètres.

M. DUBOIS. - Je voudrais faire un commentaire sur la question de $M$. Duffaut, qui répondra aussi à ce qu'a dit $M$. Luong, au début de sa conférence, sur les faits qui se sont produits à Nüremberg où, après quelques ennuis, une étude systématique a été faite sur plusieurs types de matériaux à injecter.

Dans cette étude, les laboratoires allemands ont regardé la résistance à la compression simple et la résistance à la compression confinée en fonction du temps. Le confinement peut augmenter la résistance de $50 \%, 100 \%$ et plus, dans des cas types, suivant les conditions.

D'autre part, si leurs études au laboratoire sont moins systématiques que les vôtres, elles ont permis d'évaluer l'influence du confinement, puis ont servi de base à des calculs de déformation et de stabilité, utiles pour juger l'efficacité de tel ou tel matériau à injecter.

A cet effet, nous avons mis au point avec eux un modèle rhéologique qui tient compte des propriétés du matériau à très court terme (c'est-à-dire des essais à vitesse de déformation donnée) et des propriétés à long terme (à partir d'essais de fluage à long terme).

Nous avons dû le faire relativement complet, par une série de modèles rhéologiques élémentaires comme celui que vous avez présenté, en tenant bien compte du fait qu'il fallait prendre un amortissement en logarithmes et non pas un amortissement constant, comme vous l'avez souligné.

A partir de ce modèle rhéologique, nous avons construit un modèle numérique pour simuler les essais dont je viens de parler.

Nous avons ensuite utilisé ce modèle numérique dans un calcul effectué avec la méthode des éléments finis sur des géométries représentatives de sections de galeries de métro. Nous avons trouvé que, pour certains types de matériaux que l'on injectait, la déformation à long terme n'était pas diminuée.

Pour Nüremberg, il n'y avait pas de problème de stabilité, mais des problèmes de déformation conduisant éventuellement à des fissures dans les bâtiments voisins. Nous avons regardé ce problème et mis en évidence que, pour certains matériaux, l'injection ne réduisait pas les déformations à long terme. Ce résultat ne s'applique pas à tous les matériaux.
Nous avons aussi examiné à quel endroit il fallait injecter pour optimiser le renforcement. Cette étude n'est pas terminée.

M. HABIB. - Je voudrais poser une question à $M$. Allemand. Au cours de son exposé, M. Luong nous a montré des résistances qui évoluent en fonction du temps suivant des droites en coordonnées logarithmiques. C'est désagréable parce que, si on extrapole sur un temps suffisamment long, on arrive à un moment où la résistance est nulle. Si on attend même un peu plus longtemps, elle devient même négative et cela devient franchement curieux.

En associant une résistance à une durée de travaux. ma question est la suivante : est-ce que vous pensez, en tant que chimiste, que la résistance puisse diminuer jusqu'à 0 , ou bien qu'il doive rester quelque chose? Quand on extrapole en coordonnée semi-logarithmique pendant toute la fin de l'ère quaternaire, ce n'est pas bien grave; mais quand c'est à deux ou trois ans, comme pour certaines des compositions étudiées ici, ça peut être plus ennuyeux. Or, les essais ont été faits jusqu'à deux ou trois mois: jusqu'où pensez-vous qu'on peut aller raisonnablement?

M. ALLEMAND. - Je pourrais vous répondre tout d'abord qu'il suffit d'avancer d'une décade ou deux, ce n'est pas beaucoup, sur le papier logarithmique et ca reporte chaque fois pas mal en avant, comme vous l'avez souligné.

Au niveau chimique, nous nous sommes posés la question sur l'aspect un peu plus théorique, en particulier sur la configuration possible des produits solides qui donnent la résistance mécanique qu'on a observée. On avait un espoir, c'était qu'en fabriquant un réseau chimique le plus régulier possible et qui se rapproche le plus d'un solide parfait, on obtiendrait quelque chose qui ait un comportement dans le temps le plus stable possible.

On voit, d'après les résultats qu'a mis en lumière $M$. Luong, que les résines du type acrylique ont un comportement qui semble beaucoup plus confortable que les gels de silicate. C'est une conséquence des suppositions qu'on avait faites, c'est-à-dire que lorsqu'on crée un réseau qui est régulier (et on peut représenter un gel acrylique théoriquement par un assemblage à trois dimensions, donc c'est un solide parfait) on voit qu'effectivement on a un comportement dans le temps beaucoup plus confortable.

Un gel de silicate, au contraire, peut être représenté par un assemblage extrêmement irrégulier de molécules, avec des liaisons qui ne sont pas du même ordre, de même nature que dans le gel acrylique; on voit que, dans ce cas-là, le comportement dans le temps est beaucoup moins sûr.

Je ne peux pas répondre avec certitude au problème du comportement dans le temps, mais je pense que si on veut avoir, je ne dis pas une certitude, mais disons de bonnes raisons de penser qu'on aura une bonne stabilité. il faut, soit se tourner vers les produits du type acrylique, soit s'orienter vers les gels de silicate en dilution assez forte et surtout à fort taux de neutralisation, c'està-dire que la neutralisation du silicate doit être réalisée par une quantité de réactif importante.

M. Armand MAYER. - J'ai été impressionné et très intéressé par la conférence de $M$. Luong et par le grand nombre des essais qu'il nous a décrits ; il y a tout de même un point qui m'étonne.

L'expérience prouve qu'on a fait des injections, qu'elles tiennent et qu'on n'a pas constaté de résultats du genre de ceux qu'il nous a présentés. Je me demande si le fait de s'être limité à un matériau ayant une granulométrie uniforme, comme le sable de Fontainebleau. n'est pas pour beaucoup dans ces résultats. 
II me semble qu'il serait extrêmement intéressant ae reprendre l'opération et de faire un certain nombre d'essais, les plus typiques, avec un sable ayant une granulométrie continue, c'est-à-dire partant du millimètre et descendant à des dimensions beaucoup moins fortes. Il est bien entendu qu'on n'utilise pas le silicate pour étancher ou pour consolider un matériau à gros vides. Là, on emploie le ciment et on est sûr du résultat. Si on a un matériau naturel dont la granulométrie est relativement continue, il y a des vides importants, mais il y en a aussi de beaucoup plus petits et l'expérience prouve que les résultats sont bons.

Est-ce que le fait d'injecter ces matériaux-là ne donnerait pas des résultats plus satisfaisants que ceux que vous avez obtenus sur le sable de Fontainebleau?

M. GANDAIS. - Nous avons utilisé le sable de Fontainebleau parce que de nombreuses études de laboratoire se font avec ce sable, si bien que nous disposions de points de repère et qu'il était possible de se référer à des études antérieures.

D'autre part, si on examine un sable injecté avec un produit du type gel de silicate ou résine, on se trouve devant un squelette composé de grains très durs et d'une matrice molle par comparaison avec la dureté de ces grains. Comme dans la littérature on trouve des articles qui précisent que l'angle $\varphi$ du squelette est peu affecté par l'injection de tels produits, nous avons pensé que l'étude du comportement de la matrice, que nous voulions faire, ne serait pas affectée par la granulométrie du sable utilisé.

Nous sommes bien d'accord que si nous prenons des sables différents, avec des grains plus gros ou de formes différentes, nous obtiendrons des valeurs de résistance différentes. Cependant, nous pensons que les caractères propres au comportement de la matrice ne seront pas modifiés.

M. A. MAYER. - Dans les essais que vous faites, vous comprimez votre matériau. Autrement dit, vous réduisez les vides, vous avez un matériau uniforme, il n'y a pas compactage. Si vous avez un matériau continu, il y a compactage et augmentation de la résistance. Cela me paraît évident et je serais très heureux qu'on fasse quelques essais dans ce sens, sinon on risque de tirer de votre conférence des conclusions quí ne sont pas du tout celles que vous attendez.

M. CARON. - Comme MM. Allemand et Mayer, je continue à avoir confiance dans les injections.

Esope disait que la langue est la meilleure et la pire des choses; on peut en dire autant des gels de silice, tout dépend de la composition que l'on a adopté.

Dans toutes les courbes que vous avez présentées. certaines sont très alarmantes, surtout si on les extrapole à quelques années. Mais si on regarde celles qui ont un taux de neutralisation de 0.9 , c'est alors beaucoup moins grave. Lorsque des gels de résistance instantanée de 20 à 30 bars tombent à 1 bar au bout d'un mois, c'est parce qu'ils ont été mal calculés, c'est-à-dire qu'on a utilisé un taux de neutralisation trop faible. L'injecteur aura donc à choisir, dans toutes les formules envisageables, celle qui donne à long terme le meilleur résultat. Pour diminuer cet effet fluant des gels, il faut accroître le taux de neutralisation, c'est-à-dire augmenter la quantité de durcisseur. Donc c'est avant tout une question de formulation.

On a parlé beaucoup tout à l'heure de cet incident en Allemagne. Je crois savoir qu'il s'est produit sur un chantier où il n'y avait pas d'injecteur à proprement parler : les produits avaient été achetés chez "l'épicier du coin ». Au même titre que l'orsqu'on va chez un pharmacien, il est prudent de passer d'abord par un docteur, je pense que lorsqu'on a un terrain à traiter. ou bien on passe par une entreprise spécialisée, ou bien on s'adresse à un ingénieur-conseil. II en est de très bons.

M. GANDAIS. - II est bien évident qu'en faisant cet exposé, nous, n'avions pas l'intention de faire peur. puisque nous sommes, nous-mêmes, des spécialistes injecteurs.

Je tiens seulement à apporter une précision : il est évident que si on extrapole certaines courbes, on arrive parfois à une valeur de résistance nulle. N'oublions pas que les représentations graphiques que vous avez vues sont des représentations géométriques empiriques à partir de résultats expérimentaux. De par nature, de telles courbes ne tolèrent pas l'extrapolation.

II est bon de noter également que l'évolution des phénomènes que nous avons rencontrés se représente par des droites en coordonnées semi-logarithmiques. Cela signifie que l'on a affaire à des phénomènes amortis dans le temps. II nous semble réconfortant de savoir que la variation des résistances va en s'amenuisant avec le temps. Ce qui est encore plus réconfortant est tout simplement de le savoir et de pouvoir mieux étayer nos choix.

M. DUPEUBLE. - Je voudrais rappeler nos préoccupations lorsque nous avons commencé cette étude. Elles étaient de deux ordres.

Tout d'abord ce que nous trouvions dans les cahiers des charges, lorsque nous devions répondre à un appel d'offres qui nous imposait des résistances à la compression simple, nous gênait beaucoup car nous avions conscience que cela ne signifiait pas grand chose. On sentait très nettement que I'on parlait implicitement de notions de résistance appliquées à des matériaux comme les bétons et que cela se traduisait par : " traitez ce sol pour obtenir une résistance de 2, 2.5 ou $3 \mathrm{MPa}$ *. Nous voulions donc aller plus avant pour essayer de montrer que cette notion de résistance mécanique était liée à la notion de temps.

Notre deuxième préoccupation, complètement différente mais cependant directement dérivée de la première, était que nous craignions de passer peut-être à côté d'un certain nombre de produits, de formulations rejetées jusqu'alors, parce que nous obtenions des résistances à la compression simple, suivant les critères utilisés jusqu'alors, trop faibles pour pouvoir être prises en considération par un client. Pourtant, ces mêmes produits que nous rejetions à priori pouvaient peut-être présenter à long terme une résistance pratique, utile, supérieure à celle de produits paraissant beaucoup plus brillants par une approche un peu trop rapide des phénomènes.

Nous n'avons jamais voulu faire peur à personne en lançant cette étude, mais nous avons voulu justement approfondir la connaissance des phénomènes et introduire, comme le disait tout à l'heure $\mathrm{M}$. Luong, le facteur temps et la façon dont les travaux ultérieurs de réalisation de l'ouvrage sont menés: car on s'apercoit que les deux éléments qui entrent en ligne de compte sont bien la durée de l'exécution de l'ouvrage, excavation par exemple d'un tunnel ou d'une fouille, et la façon dont est sollicité le traitement de protection de la dite excavation.

Nous avons voulu apporter une dimension supplémentaire au projeteur et élargir les possibilités de choix.

M. TCHENG. - Comme on le disait tout à l'heure, pour faire l'étude en vue de travaux de tunnel ou d'excavation, et peut-être à tort, nous avions besoin de $\varphi$ et de c. Lorsqu'il y a une décroissance de résistance à la compression simple, est-ce que c'est plutôt la cohésion qui diminue ou bien plutôt l'angle de frottement, et dans quelle proportion croyez-vous que les caractéristiques tombent?

Je voudrais également savoir si cette chute de résistance dépend de l'état de conservation de l'échantillon, avec paraffine ou sans paraffine; est-ce que cela donnerait des différences?

M. LUONG. - Notre étude concerne l'influence du facteur temps sur la résistance à la compression simple et nous l'avons réalisée sur un seul sol de référence. Nous n'avons pas fait d'essais dans l'optique d'une étude des variations éventuelles de $\varphi$ et de $c$. Cependant, on trouve, dans la littérature, I'information que lorsque $\varphi$ change l'allure des courbes reste la même. 
En ce qui concerne les essais de fluage, il faut essayer de maintenir l'échantillon dans un état identique à l'état initial. Une perte d'eau peut induire des phénomènes de forces capillaires qui risquent de modifier la résistance des éprouvettes. II nous semble donc indispensable de bien protéger les éprouvettes.

M. GANDAIS. - J'ajouterai que la conservation à l'air, qui peut entraîner une dessication, ne nous intéresse pas, puisqu'elle ne correspond pratiquement à rien dans la réalité. Par contre, cette étude que nous avons présentée ce soir n'est pas terminée et nous avons des échantillons qui ont été injectés, comme vous l'avez vu sur les photos, et qui sont actuellement conservés dans I'eau. Nous pourrions voir dans plusieurs mois comment se comporteront ces échantillons au fluage.

M. TCHENG. - Je crois que le cas d'une conservation d'échantillon sans paraffine correspond bien à une paro excavée et exposée à l'air du vendredi soir jusqu'au lundi matin.

M. LUONG. - C'est possible, mais en deux jours, je crois que l'éffet de la dessication est moins important que pour un essai qui dure plus de trois mois.

M. le PRESIDENT. - On utilise souvent des protections du terrain quand on laisse passer le week-end. Cela n'a rien à voir avec les injections.

M. CAMBEFORT, - Je voudrais savoir si on a bien vérifié que le volume de coulis injecté correspondait au volume des vides du sable, parce que s'il est supérieur les grains ont été séparés les uns des autres et en fin de compte ce n'est pas du sable injecté qu'on a, mais presque la matrice pure.

M. LUONG. - Par manque de temps, je n'ai pas décrit la technologie des colonnes pour injection. Le sable est mis en place dans un tube en matière plastique. Ce sable est maintenu par deux disques obturateurs qui empêchent son expansion pendant l'injection. D'autre part, nous avons essavé de maintenir la pression d'injection à un niveau très faible pour ne pas créer de désordre dans l'état initial du sable.

De toute façon, nous avons fait des mesures de véri- fication concernant le poids à sec humide, etc. Nous avons constaté que l'indice des vides n'a pratiquement pas varié. II est resté voisin de 0.6 comme à l'état initial.

M. POUPELLOZ. - Je voudrais poser une question sur la représentativité des essais de laboratoire, notamment pour des problèmes d'injection de consolidation sous des ouvrages d'art.

En laboratoire, on réalise une imprégnation parfaite de l'échantillon; ne pensez-vous pas qu'une grande partie de la consolidation résiduelle à long terme du terrain est obtenue par claquages ou microclaquages?

M. GANDAIS. - Votre question se rapporte davantage à la technique de l'injection et à la définition géométrique de l'ouvrage à réaliser.

$\mathrm{Si}$ on a affaire à des sables fins, ou très fins, on va prévoir notre traitement de façon à se mettre dans les meilleures conditions possibles pour pouvoir procéder à cette imprégnation. Que cette imprégnation soit imparfaite est presque évident à cause de l'hétérogénéité inhérente aux terrains.

II se peut donc qu'il se produise des claquages; mais je ne pense pas que de tels claquages, réalisés avec des gels de silicate ou des résines acrylamides, apportent grand chose au niveau de la consolidation de l'ensemble de la masse traitée. Au pire, s'ils sont trop importants, ils peuvent être nuisibles parce qu'ils sont constitués de produits peu résistants. La technique et la conduite de l'injection doivent être menées pour éviter, ou du moins minimiser, leur formation.

M. le PRESIDENT. - Je voudrais dire qu'en tant que maître d'œuvre, j'apprécie beaucoup qu'on abandonne un peu le seul aspect de la résistance à la compression. D'ailleurs, dans beaucoup de domaines, ce critère s'avère insuffisant pour caractériser une roche ou un sol. J'apprécie aussi que, pour la définition d'un ouvrage et pour sa protection, on puisse dégager des facteurs dont l'influence est toujours très importante, ainsi que vous nous l'avez montré.

II nous reste donc à vous remercier de nous avoir exposé les résultats de cette très intéressante étude. 
